\title{
Solar Temperature Variations Computed from SORCE SIM Irradiances Observed During 2003 - 2020
}

\author{
Robert F. Cahalan ${ }^{1}$ (D) Paulino Ajiquichí ${ }^{2}$. Gaspar Yatáz ${ }^{2}$
}

Received: 19 October 2021 / Accepted: 17 December 2021 / Published online: 27 January 2022

(C) The Author(s) 2022

\begin{abstract}
NASA's Solar Radiation and Climate Experiment (SORCE) Spectral Irradiance Monitor (SIM) instrument produced about 17 years of daily average Spectral Solar Irradiance (SSI) data for wavelengths $240-2416 \mathrm{~nm}$. We choose a day of minimal solar activity, August 24, 2008 (2008-08-24), during the 2008-2009 minimum between Cycles 23 and 24, and compute the brightness temperature ( $\left.T_{o}\right)$ from that day's solar spectral irradiance $\left(S S I_{o}\right)$. We consider small variations of $T$ and $S S I$ about these reference values, and derive linear and quadratic analytic approximations by Taylor expansion about the reference-day values. To determine the approximation accuracy, we compare to the exact brightness temperatures $T$ computed from the Planck spectrum, by solving analytically for $T$, or equivalent root finding in Wolfram Mathematica. We find that the linear analytic approximation overestimates, while the quadratic underestimates, the exact result. This motivates the search for statistical "fit" models "in between" the two analytic models, with minimum root-mean-square-error, RMSE. We make this search using open-source statistical $R$ software, determine coefficients for linear and quadratic fit models, and compare statistical with analytic RMSEs. When only linear analytic and fit models are compared, the fit model is superior at ultraviolet, visible, and near-infrared wavelengths. This again holds true when comparing only quadratic models. Quadratic is superior to linear for both analytic and statistical models, and statistical fits give the smallest RMSEs. Lastly, we use linear analytic and fit models to find an interpolating function in wavelength, useful when the SIM results need adjustment to another choice of wavelengths, to compare or extend to any other instrument. Advantages of the quadratic $T$ over the exact $T$ include ease of interpretation, and computational speed.
\end{abstract}

Keywords SORCE · Irradiance · Temperature · Linear approximation · Quadratic approximation

This article belongs to the Topical Collection:

The Solar Radiation and Climate Experiment (SORCE) Mission: Final Calibrations and Data Products Guest Editor: Thomas N. Woods

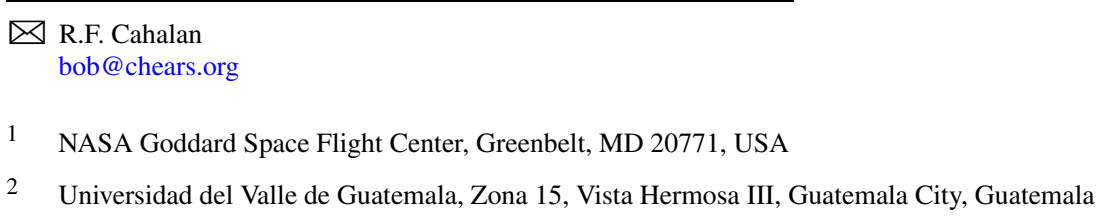




\section{Introduction}

The Sun's temperature and its variations over timescales from hours to decades have been determined since 1978 from satellite measurements of associated variations in Total Solar Irradiance (TSI). Since the deployment of the SORCE satellite in 2003, the Sun's temperature has also been determined for a continuous range of wavelengths that span ultraviolet, visible and near-infrared wavelengths, from solar spectral irradiance (SSI) measurements across the peak of the SSI distribution. These are of great interest due to the fundamental role that solar variations play in understanding the variations of the Earth's climate (Harder et al., 2005; Eddy, 2009). Beyond decadal timescales, the solar irradiance is key to estimating the Sun's luminosity, and on the longest timescales it determines Earth's lifetime, since it determines when the Sun will exhaust its energy from fusion of hydrogen in the core (Bahcall, 2000).

The average radiative temperature of the Earth is determined by an approximate balance between the amount of energy it receives from the Sun, which can be calculated from the TSI and the Earth's albedo, and the amount of energy that Earth emits into space that depends on Earth's emissivity (Stephens et al., 2015). Earth's albedo is the fraction of solar energy reflected back into space, which averages about $30 \%$, the remainder being absorbed by the atmosphere and surface (Wild et al., 2013). To determine how solar variations impact Earth's atmosphere-ocean system at various heights, SSI must be monitored in addition to TSI.

The relationship between Earth's temperature and the variability of solar irradiance was first speculated on by Herschel, and as observations have improved, so has the understanding of solar variability and its contribution to climate change (Gray et al., 2010; Bahcall, 2000). The variability of both TSI and SSI occurs due to variations in magnetic fields on the solar surface, which in turn cause the appearance of sunspots and faculae (Shapiro et al., 2015). Various models attempt to predict these changes over a wide range of timescales.

Prior to SORCE, limited observations of SSI made studying the variability of SSI and solar brightness temperature difficult, but databases over several years now enable these calculations that are important for both Heliospheric and Earth sciences (Rottman and Cahalan, 2002). In this article, we present a study of linear and quadratic analytic and statistical approximations of the solar brightness temperature, $T$, using either a single "reference day" during a solar minimum, or using statistical properties over many days in the available record. The estimation of values using linear and quadratic approximations, both analytic and statistical, are of great help in simplifying the calculations of $T$, and in interpreting its variability. In order to determine the accuracy of the approximated values, it is necessary to compare them with very nearly exact values of $T$, calculated from the monochromatic exact analytic $T$ equation, Equation D.2, derived from the Planck distribution D.1, or equivalently by applying root-finding techniques to the Equation D.3 that implicitly determines $T$ from observed values of SSI.

This paper shows that the daily values of $T$ over the SIM wavelengths are well determined from a polynomial that is quadratic in the observed daily values of SSI. The coefficients may be expressed as analytic functions of wavelength, with small RMS errors. Even smaller RMS errors are achieved with coefficients determined from statistical fits of the observed data. Advantages of the quadratic $T$ over the exact $T$ include ease of interpretation, and computational speed. Interpretation of the linear term is the sensitivity of $T$ to changes in SSI, a concept widely used in climate studies. The speed gain will become important as time-dependent models of solar variations are further developed.

The article is structured as follows: Section 2 describes the data analyzed here, provides the online link to download it, and describes the temporal and wavelength range. Section 3 summarizes the methodology used for the analysis of the SSI spectral data, the calculation of exact values of solar brightness temperatures $T$, as well as linear and quadratic 
analytic and statistical approximations of $T$. Section 4 discusses the results of exact computations of $T$, the time series of observed SSI, and the time-series comparisons of exact and approximate $T$ values. Section 5 concludes by summarizing the key results and suggests future directions for research related to variations in solar irradiance and brightness temperature. Finally, the article contains nine appendices that derive results referenced in Sections 1-5. Several figures and tables are discussed throughout. Readers may interact with several of the plotted results by going to the following dashboard that was coded in Microsoft Power BI. Dashboard link: http://wayib.org/solar-temperature-variations-relative-toa-quiet-sun-day-in-august-2008/.

\section{Data}

The TSI and SSI data were downloaded from the University of Colorado's LASP Interactive Solar Irradiance Datacenter (LISIRD), based on measurements made by instruments onboard the Solar Radiation and Climate Experiment (SORCE) satellite. The data is free and publicly available here: https://lasp.colorado.edu/lisird/data/sorce_sim_ssi_13/.

The SORCE Total Irradiance Monitor (TIM) instrument provides records of Total Solar Irradiance (TSI), while the Spectral Irradiance Monitor (SIM) instrument provides records of the Solar Spectral Irradiance (SSI). Both instruments provide daily averages, with TIM beginning 2003-02-25, and SIM beginning 2003-04-14 and both ending on 2020-02-25 when the SORCE instruments were passivated (i.e., turned off). We employ throughout the latest "final" data versions, v19 for TSI, and v27 for SSI, as discussed in Kopp (2020) and Harder (2020), respectively. All dates in this article are given in the format YYYY-MM-DD, in accord with https://www.iau.org/static/publications/stylemanual1989.pdf.

The SIM measures SSI as a function of wavelength over the range from $240 \mathrm{~nm}$ to $2416 \mathrm{~nm}$. Though measurements of SSI were made prior to SORCE, for example by the UARS SOLSTICE (operating during 1991-2001), SIM was the first to provide SSI for a continuous range of wavelengths across the peak of the solar spectrum that occurs near $500 \mathrm{~nm}$, and well into the near-infrared (IR) wavelengths, with sufficient precision to determine true solar variations (see, e.g., Harder et al., 2009; Lee, Cahalan, and Dong, 2016).

Note that all irradiance data from SORCE, including all TSI and SSI values, are adjusted to the mean Earth-Sun distance of one astronomical unit, 1 AU. Doppler corrections are also made to remove any variations due to the satellite orbit. Absolute and relative calibrations are enabled by a variety of laboratory measurements carried out at both the University of Colorado's LASP (Laboratory for Atmospheric and Space Physics), and at NIST facilities. Onboard instrument degradation is monitored and corrected for. Our focus in this paper is on the day-to-day variability at near-ultraviolet, visible, and near-infrared wavelengths. For this, we rely primarily on the high precision and repeatability of TIM and SIM, more than on the absolute calibration. The high quality of TIM and SIM data has been amply documented in the literature.

Due to operational difficulties encountered, particularly after 2011 as SORCE aged, there are a limited number of days where the records are given as NA (not available) or no values were recorded. These were omitted in all calculations reported here. As an example of the SSI records measured by the SIM instrument, the time series of the solar spectrum from 2003 to 2020 is shown in Figure 4 for a fixed wavelength, $656.20 \mathrm{~nm}$, which corresponds to the hydrogen alpha $(\mathrm{H} \alpha)$ transition in the Balmer series.

For much of the data analysis, open-source $R$ and Python software was used, as well as commercial software including Wolfram Mathematica, and Microsoft Excel. Mathematica 
enabled precise computation of the brightness temperatures of the SSI data, using efficient interpolation and root-finding methods, and provided a check on exact values computed from the analytic equation for $T$ derived from the Planck distribution for the spectral irradiance, shown in Appendix D.

For more details on the TSI and SSI data used here, see the "release notes" for SORCE TIM v19, and for SORCE SIM v27, available from the NASA Goddard Space Flight Center Earth Sciences Data and Information Services Center, or from the University of Colorado's LASP (Harder, 2020; Kopp, 2020).

\section{Methodology}

For the radiation from a blackbody, the irradiance spectrum may be computed theoretically using the Planck distribution. However, the Sun is not a perfect blackbody, due to wavelength-dependent processes in the Sun's atmosphere. Large deviations from the Planck distribution are observed, as we show below. However, it is very useful for interpreting irradiance observations to define a solar "brightness temperature," either for the TSI, integrating all wavelengths, or for the solar spectral irradiance, SSI, at each available wavelength. This is the temperature for which the irradiance computed from a Planck distribution coincides with the irradiance observed by an instrument outside Earth's atmosphere, for example TIM for the wavelength-integrated irradiance, the TSI, or SIM for the wavelength spectrum of irradiance, SSI.

Computation of the brightness temperature from TSI, $T_{\text {eff }}$, is simply a matter of explicitly solving the Stefan-Boltzmann Law for $T_{\text {eff }}$, with a result proportional to the one-quarter power of TSI. Appendices A, B and C discuss the importance of TSI and related quantities. Appendix D displays the Equations D.2 and D.3 that determine the value of the spectral brightness temperature $T$ as an explicit function of the observed SSI for each fixed wavelength. Equations D.1 and D.3 also determine $T$ as an implicit function of the observed SSI, by solving Equation D.3 for $T$ as a function of SSI at each fixed wavelength using a rootfinding procedure. We employ a root-finding algorithm developed in Wolfram Mathematica, using the following initial condition $T=5770 \mathrm{~K}$, where $T$ is chosen near the effective radiative temperature computed using $T S I=1360.8 \mathrm{~W} / \mathrm{m}^{2}$ as provided by the SORCE TIM (Kopp and Lean, 2011). These two approaches produce the same values of $T$, referred to in this paper as the "exact" values, and each method provides a check on the other.

SORCE SIM provides a daily SSI record for each associated wavelength from $240 \mathrm{~nm}$ to $2416 \mathrm{~nm}$, so that over the 17 year period there is a large amount of data. To handle the large number of records, algorithms were developed in R, Python and Mathematica, to provide approximate values of $T$. These approximate alternatives allow more rapidly computed values of $T$ for any date, given a fixed set of wavelengths. In this article, we investigate linear and quadratic analytic approximations as a function of the observed SSI values, derived in Appendix E. Below, it will be shown that these approximations bracket the exact values, which motivates the development of linear and quadratic fit approximations, that minimize the root-mean-square-error (RMSE) across a large range of days, which can include all available days. These fit approximations are developed in Appendix G.

For the development of the linear and quadratic analytic approximations, a Taylor expansion is used (see Appendix E). Having the derivatives of $T$ with respect to the SSI, this expansion gives a representation of $T$ in terms of polynomial functions of SSI. To keep the models simple, only the first and second terms of this expansion are considered. 
To apply a Taylor expansion it is necessary to have a reference value around which to expand. For this, we choose the SSI on a single "reference" day during the 2008-2009 solar minimum of Cycle 23. Namely, we choose 2008-08-24, and label that day's exact values $\left(T_{o}, S S I_{o}\right)$. With the observed value of $S S I_{o}$ and associated computed value of $T_{o}$ during a solar minimum, the linear and quadratic coefficients were calculated for the analytic approximation models. The remainder of this section discusses the time series of SSI and estimated $T$ values. Section 4 then compares the approximate values with the exact values, and also compares the analytic approximations with analogous fit approximations that use coefficients obtained by minimizing RMSE (root-mean-square-errors) over all days, and also over two selected ranges of days.

To compare the estimation with the exact value of brightness temperature, we compute difference values, and relative differences, or delta values as:

$$
\text { Delta }=\frac{T_{\text {exact }}-T_{\text {estimate }}}{T_{\text {exact }}} .
$$

In addition to the linear and quadratic analytic approximations obtained with the Taylor expansion, a linear and quadratic fit model is developed in Appendix G, with the help of $R$ statistical software. The linear and quadratic fit models have coefficients that depend on a given temporal range of available data, and not only on the chosen reference day, as is the case with the analytic approximations. In Section 4 we report results for the full range of available days, as well as for two subranges, those of "early" and "late" days, R1 and R2, respectively.

The comparison between the brightness temperatures calculated with the linear and fit approximations are shown in the tables, along with a comparison between the linear coefficients. In order to make the computations very explicit, in Appendix H, an example of the calculation of the brightness temperature is given for the linear and quadratic analytic approximation methods, as well as for the linear and quadratic fit approximation methods, for a randomly selected day.

In Appendix I, a method of rapid interpolation is given for the linear analytic and fit coefficients, valid over a broad range of wavelengths that satisfies $400 \mathrm{~nm} \leq \lambda \leq 1800 \mathrm{~nm}$.

\section{Results}

Before considering the temporal variations of SSI observed by the SORCE TIM and SIM instruments over the 17 years, 2003 -2020, we first consider the wavelength variations of SSI on our chosen "reference day" 2008-08-24. Figure 1 shows this $S S I_{o}$ wavelength dependence observed on the reference day, in green, and for comparison the Planck irradiance distributions computed for temperatures $T=4500 \mathrm{~K}, 5770 \mathrm{~K}, 6500 \mathrm{~K}$ using Equation D.1, in blue, tan, and red, respectively. The lower and upper Planck temperatures are seen to give computed $S S I$ values that bracket the observations of $S S I_{0}$ for this wavelength range, while the computed $S S I$ for the intermediate $5770 \mathrm{~K}(\tan )$ approximately follows the observed $S S I_{o}$ (green). Although the observed value coincides with the computed $5770 \mathrm{~K}$ Planck value for a few wavelengths only, the observed values of $S S I_{o}$ lie above or below the Planck curve. The measured value of TSI (historically "solar constant") by the Total Irradiance Monitor (TIM) instrument on the reference day is $T S I_{o}=1360.4704 \mathrm{~W} / \mathrm{m}^{2}$ and is associated with an effective radiative temperature of $T_{o}=5771.2685 \mathrm{~K}$, close to $T=5770 \mathrm{~K}$, used in computing the intermediate tan curve in Figure 1 (Kopp and Lean, 2011). 


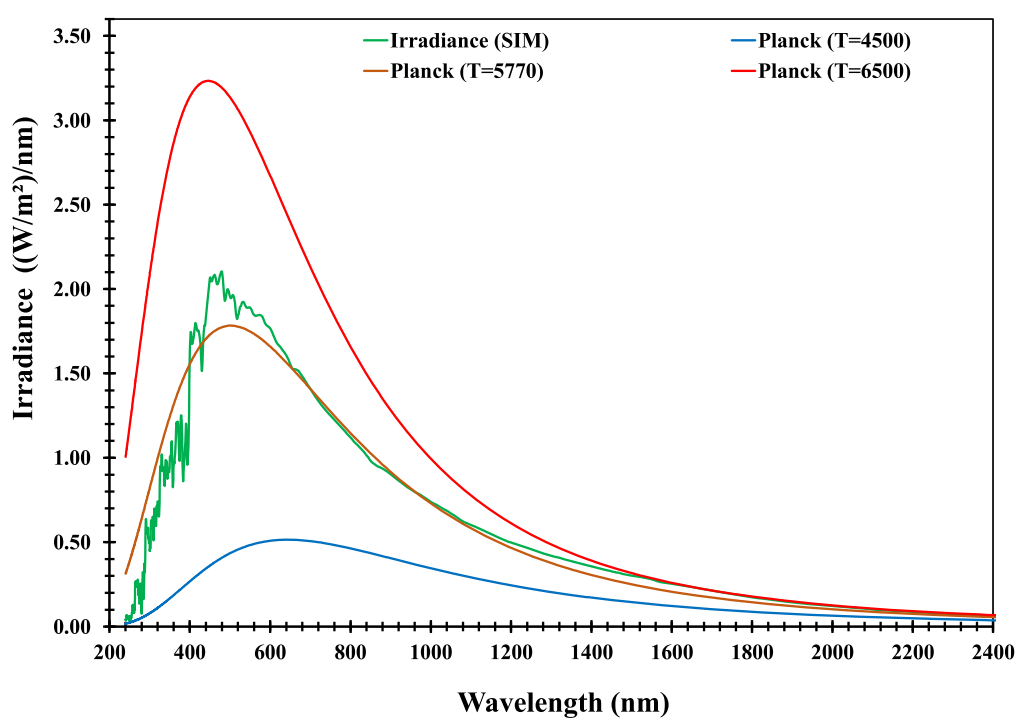

Figure 1 Solar Spectral Irradiance (SSI) vs. wavelength for reference day 2008-08-24, plotted in green, as measured by the SIM instrument onboard SORCE. For comparison, we also show Planck distributions for $6500 \mathrm{~K}$ in red, $5770 \mathrm{~K}$ in tan, and $4500 \mathrm{~K}$ in blue. The Planck distributions use Equation D. 1 for a fixed temperature, with wavelength as the independent variable, and transformed to spectral irradiance by multiplying by the factor $\alpha_{s}=\pi *\left(\frac{R_{S}}{\mathrm{AU}}\right)^{2}=6.79426 * 10^{-5}$, with $R_{S}$ the Sun's mean radius, and AU the mean Earth-Sun distance, as in Equation D.3.

Figure 2 is a zoom of Figure 1 for the wavelength range $240 \mathrm{~nm}$ to $680 \mathrm{~nm}$. The apparently irregular bumps in this plot, and in Figure 1, are due to well-known Fraunhofer lines in the solar spectrum, smoothed to the SIM instrument's bandpass, which varies from about $1 \mathrm{~nm}$ width near wavelength $240 \mathrm{~nm}$, up to almost $30 \mathrm{~nm}$ near $1000 \mathrm{~nm}$, then decreases slightly (Harder et al., 2005). The width of a typical atomic Fraunhofer line is of order $1 \AA$, or $0.1 \mathrm{~nm}$, so the observed bumps are smoothed clusters of several nearby lines. A few of the contributing atomic lines are indicated in the labels on the vertical dashed lines. For example, the green dashed line near $430 \mathrm{~nm}$, is labeled CaFeg to indicate that lines of calcium, iron, and oxygen (g-band) are all included within the plotted bump in the green line. For identification of g-band lines (both atomic and molecular) and its variability related to magnetic field strength, see Shelyag et al. (2004).

Effects of ionization thresholds are also seen, such as just above the $\mathrm{Ca}$ II $\mathrm{H}$ and $\mathrm{K}$ lines near $400 \mathrm{~nm}$, which has photon energies near $3.1 \mathrm{eV}$.

TSI provides key observational data about the Sun and is needed to compute the Sun's luminosity and lifetime (see Appendix B). TSI is not a solar constant, as had been assumed prior to the satellite era. Its value varies due to turbulent magnetic processes on the Sun. TSI variations amount to about $0.1 \%(1000 \mathrm{ppm})$ of the mean value over the four solar cycles so far observed by satellite (Cycles 21 through 24), since 1978. The average solar luminosity, and thus the TSI, is determined by nuclear processes in the Sun's core. These change over a much longer timescale than the solar cycle, up to billions of years, as nuclear processes transform hydrogen into helium. The present value of TSI, and thus solar luminosity can provide a good estimate of the Sun's lifetime, and thus the time that the Sun's nuclear fuel will eventually run out. Such calculations are shown in Appendix B, where it is shown that the current best TSI value at solar minimum, $1360.80 \pm 0.50 \mathrm{~W} / \mathrm{m}^{2}$ (Kopp and Lean, 


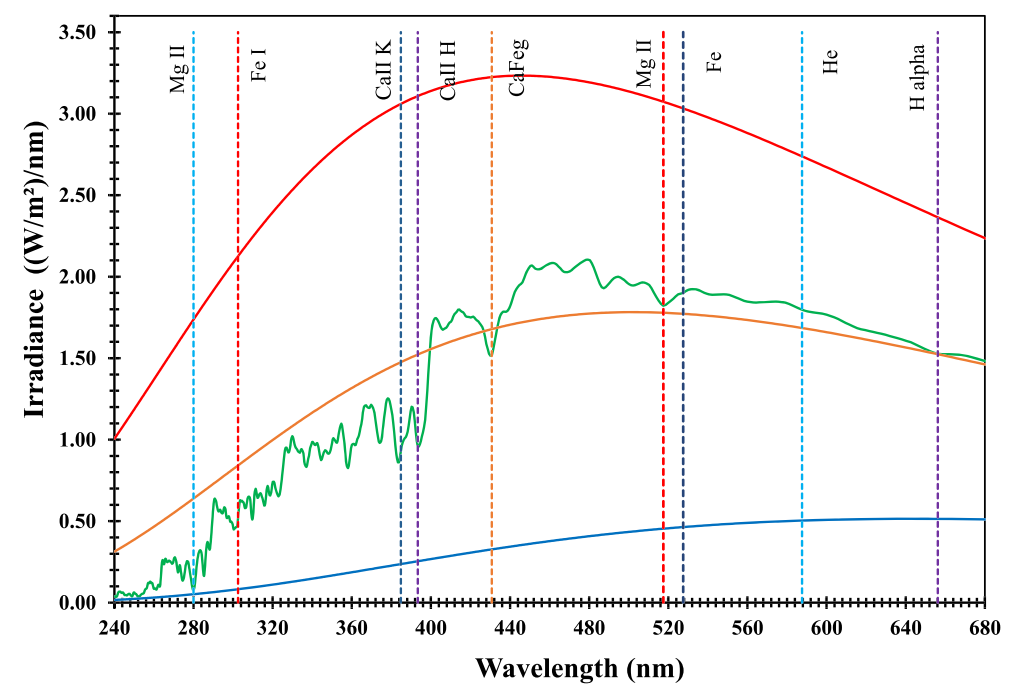

Figure 2 A zoom of Figure 1 for the wavelength range $240 \mathrm{~nm}$ to $680 \mathrm{~nm}$. The apparently irregular bumps in this plot, and in Figure 1, are due to well-known Fraunhofer lines in the solar spectrum, smoothed to the SIM instrument's bandpass, which varies from about $1 \mathrm{~nm}$ width near wavelength $240 \mathrm{~nm}$, up to about $30 \mathrm{~nm}$ width near $1000 \mathrm{~nm}$, then decreases slightly. The width of a typical atomic Fraunhofer line is of order $1 \AA$, or $0.1 \mathrm{~nm}$, so many of the observed bumps are smoothed clusters of nearby lines. A few of the contributing atomic lines are indicated in the labels on the vertical dashed lines. For example, the tan dashed line near $430 \mathrm{~nm}$, is labeled CaFeg to indicate that lines of calcium, iron, and oxygen (g-band) are all included within the plotted bump in the green line. Effects of ionization thresholds are also seen, such as just above the Ca II $\mathrm{H}$ and $\mathrm{K}$ lines near $400 \mathrm{~nm}$, which has photon energies near $3.1 \mathrm{eV}$.

2011), gives the overall lifetime of the Sun as approximately 10.70 billion years. The current estimated age of the Sun, and of our solar system, is about equal to Earth's estimated age of 4.54 billion years ( \pm 50 million years). Hence, this leaves about 6.2 billion years, more or less, before the Sun will expand into a Red Giant, leaving a white dwarf star behind.

The importance of TSI in climatic variability has been mentioned, for example in computing Earth's global average effective radiative temperature. Appendix $C$ estimates the effective temperature of the Earth as $255.48 \mathrm{~K}$, using the TSI on the reference day, and Earth's average albedo of 0.29 (Stephens et al., 2015).

TSI is the integral of SSI over all wavelengths, and SSI in turn determines the solar spectral brightness temperature $T$ at each wavelength. Determining $T$ as well as $S S I$ is useful in understanding the physical and chemical processes that take place on the Sun. For example, Figure $3 \mathrm{a}$, a plot of the brightness temperature, $T_{o}$, on the reference day, shows a broad peak above $1600 \mathrm{~nm}$. This is associated with transitions in hydrogen ions $\mathrm{H}^{-}(1$ proton +2 electrons). Photons with a wavelength $\lambda<1644 \mathrm{~nm}$ are dominated by the $\mathrm{H}^{-}$ bound-free transitions, while photons with $\lambda>1644 \mathrm{~nm}$ are absorbed and re-emitted in $\mathrm{H}^{-}$ free-free transitions (Wildt, 1939). The $\mathrm{H}^{-}$ion is the major source of optical opacity in the Sun's atmosphere, and thus the main source of visible light for the Sun and similar stars.

Now, we consider the temporal variations of SSI. Figure 4 shows the time series of the irradiance corresponding to a fixed wavelength, in particular for $\mathrm{H} \alpha(\mathrm{H} \alpha$ wavelength $=$ $656.2 \mathrm{~nm}$ ), the longest wavelength in hydrogen's Balmer Series. The variability of the SSI can be seen, with the deepest minimum occurring early in the record, during Oct-Nov 2003. The spike that goes below $1.523 \mathrm{~W} / \mathrm{m}^{2} / \mathrm{nm}$ is associated with the Halloween solar storms, a series of solar flares and coronal mass ejections that occurred from mid-October to early 

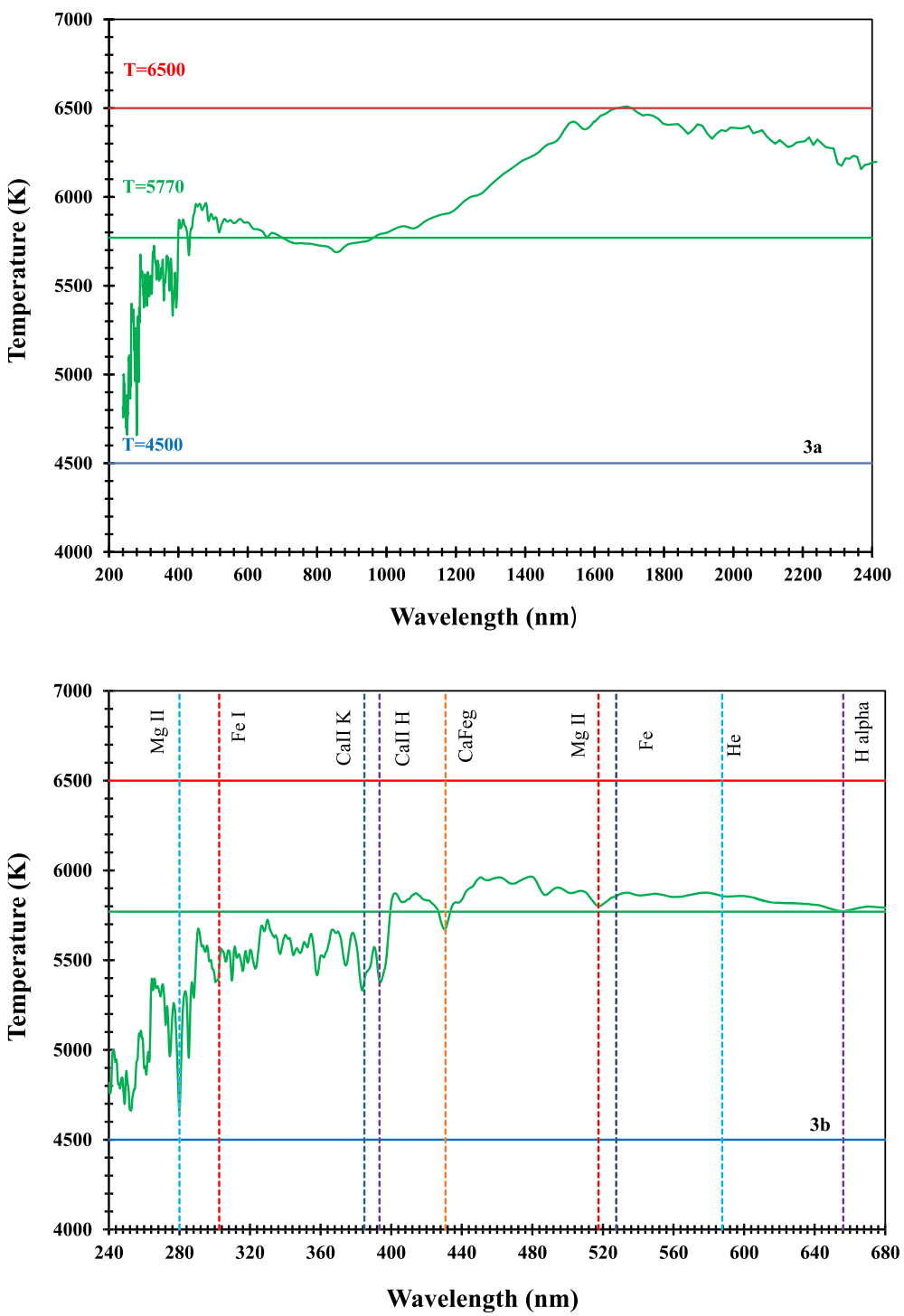

Figure 3 Temperature vs. Wavelength on the reference day, 2008-08-24. The plot in 3a shows the same wavelength range as in Figure 1. Plot $\mathbf{3 b}$ is a zoom into the same short-wavelength range as in Figure 2. As in Figure 2, several bumps are labeled with contributing atomic lines, such as the green dashed line near $430 \mathrm{~nm}$, labeled CaFeg (calcium, iron, oxygen g-band). As in Figure 2, the rise due to the ionization threshold is evident near $400 \mathrm{~nm}$, just above the $\mathrm{Ca}$ II $\mathrm{H}$ and $\mathrm{K}$ lines. In both plots, the temperature at each wavelength was computed using a Mathematica root-finding procedure to solve for $T$ in Equation D.3, $S S I=\alpha_{S} B(\lambda, T)$, with SSI the observed value.

November 2003, peaking around October 28-29. See, for example, https://en.wikipedia. org/wiki/Halloween_solar_storms,_2003.

This occurred during the declining phase of Solar Cycle 23. On the slower year-to-year timescale, the Sun's activity declines into the much quieter period of the solar minimum during 2008-2009 (Kopp, 2016). The solar minimum implies about a 0.1\% decrease in 


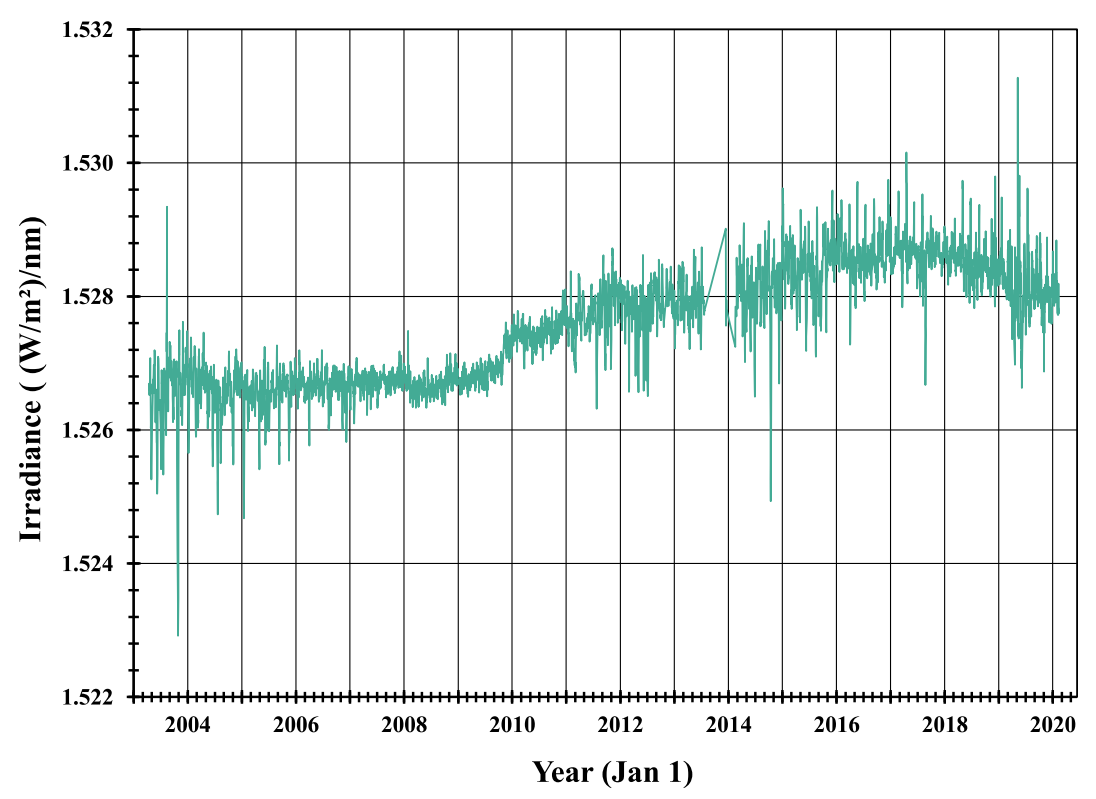

Figure 4 Time series of irradiance for all records of daily average data from the full 17 years of SIM data, version 27, downloaded from LISIRD. In this case, we have chosen the $\mathrm{H}_{\alpha}$ wavelength, $656.2 \mathrm{~nm}$. In this plot, it is evident that there is a minimum of solar activity in mid-2008. We choose as a reference day 2008-08-24, and consider variations about this day to approximate the temperatures on all other days.

solar energy that arrives on Earth, causing the Earth's temperature to decrease slightly (Gray et al., 2010). After this solar minimum, solar activity increases again, as Cycle 24 sunspots and other solar activity increase in intensity into a solar maximum in 2014-2015, before declining again, into a quieter minimum period of 2019-2020.

As can be seen in Figure 5, the brightness-temperature time series for $\mathrm{H} \alpha$ is also similar to the temporal variability of the $S S I$ for $\mathrm{H} \alpha$. It is evident that they are in phase. As $S S I$ data is extended beyond the end of SIM, by TSIS-1 and successor missions, the solar cycles will become more evident, as happened with TSI (Solanki, Krivova, and Haigh, 2013). It is important to emphasize that the spectral brightness temperatures are wavelength-dependent radiative temperatures of the Sun, the temperatures at which the SSI data measured by the satellite coincides with what is obtained using the Planck distribution (Trishchenko, 2005).

Figure 6 shows a plot of the linear analytic approximation of brightness temperature compared with the exact value, the value obtained by Equation D.2, or the root-finding solution of Equations D.1 and D.3. The linear analytic approximation is given by neglecting the quadratic term in Equation E.18, taking as reference the date during the solar minimum, 2008-08-24. Figure 6 shows that this approximation closely overlays the exact.

To more clearly see the difference between the exact and the linear analytic approximation, Figure 7a shows the difference, exact - approximation, in units of $\mathrm{mK}=10^{-3} \mathrm{~K}$, and Figure $7 \mathrm{~b}$ the delta, difference/exact (Equation 3.1) in parts per million (ppm). The negative differences in Figures $7 \mathrm{a}$ and $\mathrm{b}$ show that the linear analytic approximation overestimates the exact value of the brightness temperature. The root-mean-square-error (RMSE) is $412.4545 \times 10^{-6}$, i.e., very small, which explains why such differences are not evident in Figure 6. A significant increase in variability is seen in 2011 and afterwards, hence Figure 7a also displays the RMSE for both the earlier, quieter period, as well as the later, noisier 


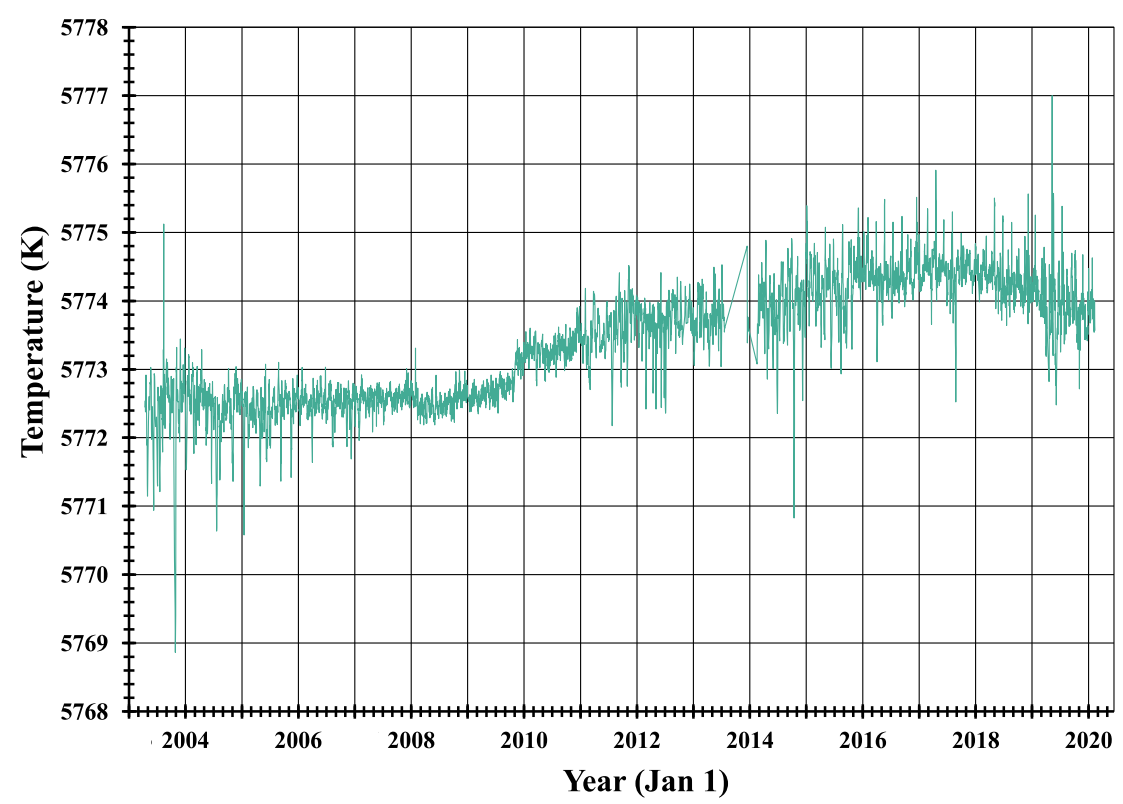

Figure 5 Time series of the temperature $T$ calculated in Wolfram Mathematica for all records of solar spectral data with fixed wavelength, $\mathrm{H} \alpha=656.20 \mathrm{~nm}$, using Equation D.3. We term the root-finding solution of Equation D. 3 the "exact" value of the temperature, to distinguish it from the two analytic approximations (linear and quadratic) described in Equation E.18, with E.10 and E.17, and from the two statistical "fit" approximations (also linear and quadratic) described in Appendix G.

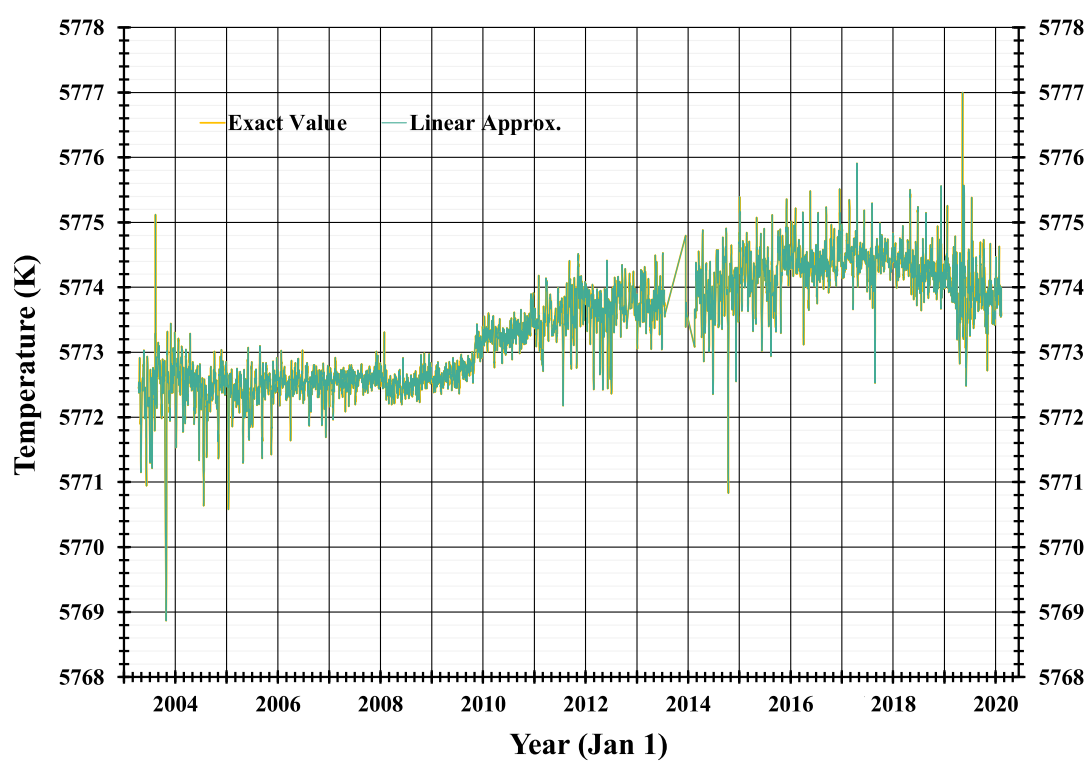

Figure 6 Linear analytic approximation of the temperature for $\mathrm{H} \alpha=656.20 \mathrm{~nm}$, compared with the exact value (value obtained by the root-finding solution of Equation D3. The linear analytic approximation is given by Equation E.18, with E.10. 

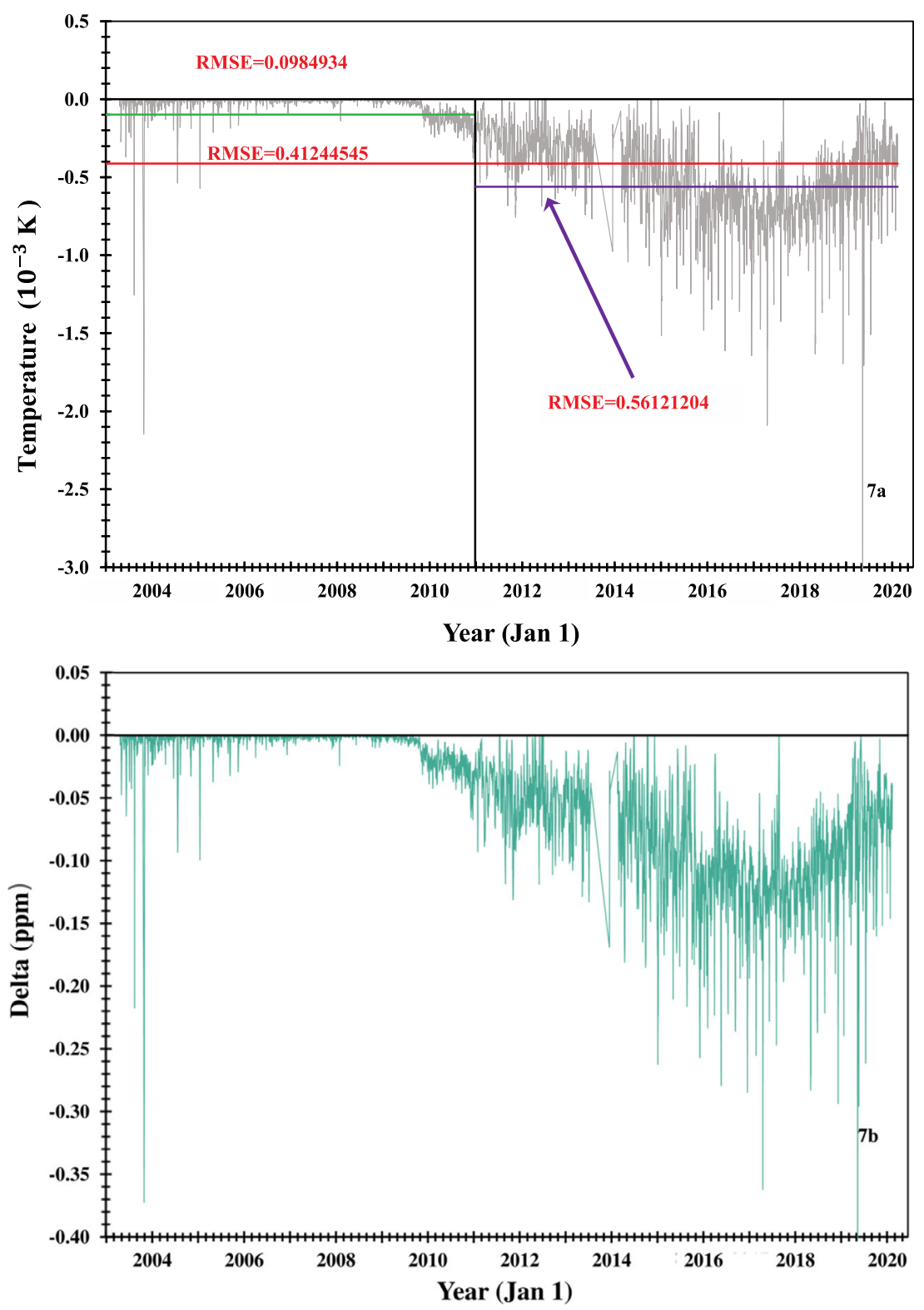

Figure 7 (a) The difference between the exact value of $T$ and the value obtained with the linear analytic approximation. (b) Delta linear approximation of the temperature using Equation 3.1 and expressed in parts per million ( $\mathrm{ppm}$ ). The negative values in this figure indicate that the linear analytic approximation overestimates the exact value of the temperature.

period. Some of this increased noise is due to Solar Cycle 24, but some is likely also due to the aging of the satellite and the SIM instrument.

Figure 8 shows the plot obtained using the quadratic analytic approximation given by Equation E.18, with D.3, together with the exact values calculated from Equation D.2 or 


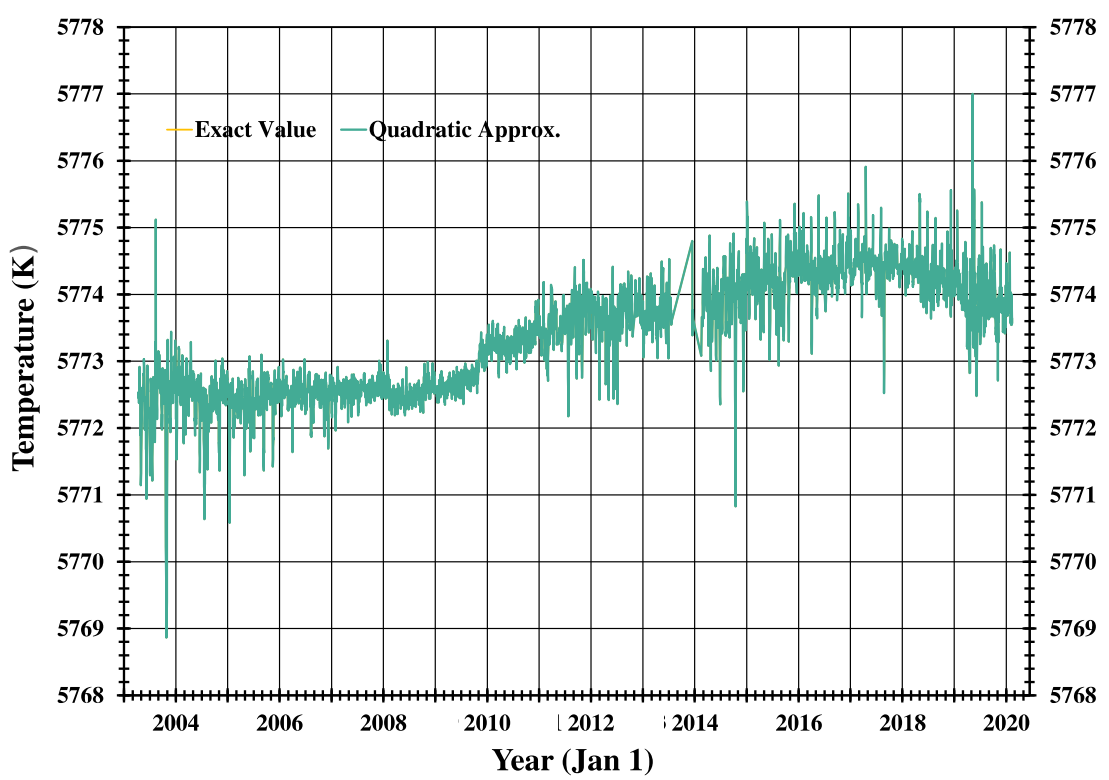

Figure 8 Quadratic analytic approximation of $T$ compared with the exact value. The quadratic approximation model is given by Equation E.18, with E.17.

from root finding with D.1 and D.3. This looks nearly identical to the analogous Figure 6 for the linear analytic approximation. However, in the plot analogous to Figure 7, we plot in Figure 9 the difference between the exact and the quadratic analytic approximation, and here the results are quite different from the linear case. Figure 9a shows the difference, exact - approximation in units of $\mu \mathrm{K}=10^{-6} \mathrm{~K}$, and Figure $9 \mathrm{~b}$ the delta, difference/exact in parts per million (ppm) in the quadratic case. The positive differences in Figures 9a and $\mathrm{b}$ show that the quadratic approximation underestimates the exact value of the brightness temperature, though are much closer than the linear, with RMSE reduced to $0.3428 \times 10^{-6}$, more than $1000 \times$ smaller than the linear case in Figure 7, and Table 2 shows that the Mean Error (Bias) is also more than $1000 \times$ smaller than the linear. Comparing Figures 7 and 9 (and Table 2) indicates that the opposite signs of the bias suggests there may be a better approximation that lies "in between" the linear and quadratic approximations. Below, we will show that the "fit" approximations do typically provide such improvements.

Figure 9a also shows that, in accord with intuition, the decrease in RMSE indicates that the approximate value is better the more terms are considered in the Taylor expansion. The improvement from RMSE in Figures 7a to 9a removes the most significant figures in RMSE in Figure 7a, suggesting a rapidly converging series. This indicates that finding an improved "in between" fit approximation will be a challenge, as the quadratic analytic approximation is excellent.

Table 2 supports this last point, comparing the RMSE for linear and quadratic analytic models, with the RMSE for the linear and quadratic fit models for the same $\mathrm{H} \alpha$ wavelength used in Figures 7 and 9. Indeed, though the linear fit model RMSE is about $2.85 \times$ smaller than the linear analytic RMSE, the quadratic fit model RMSE is $2.81 \times$ times smaller again than the $1000 \times$ smaller quadratic analytic RMSE. Hence, at the $\mathrm{H} \alpha$ wavelength, the quadratic fit model is more precise even than the very precise quadratic analytic model. 

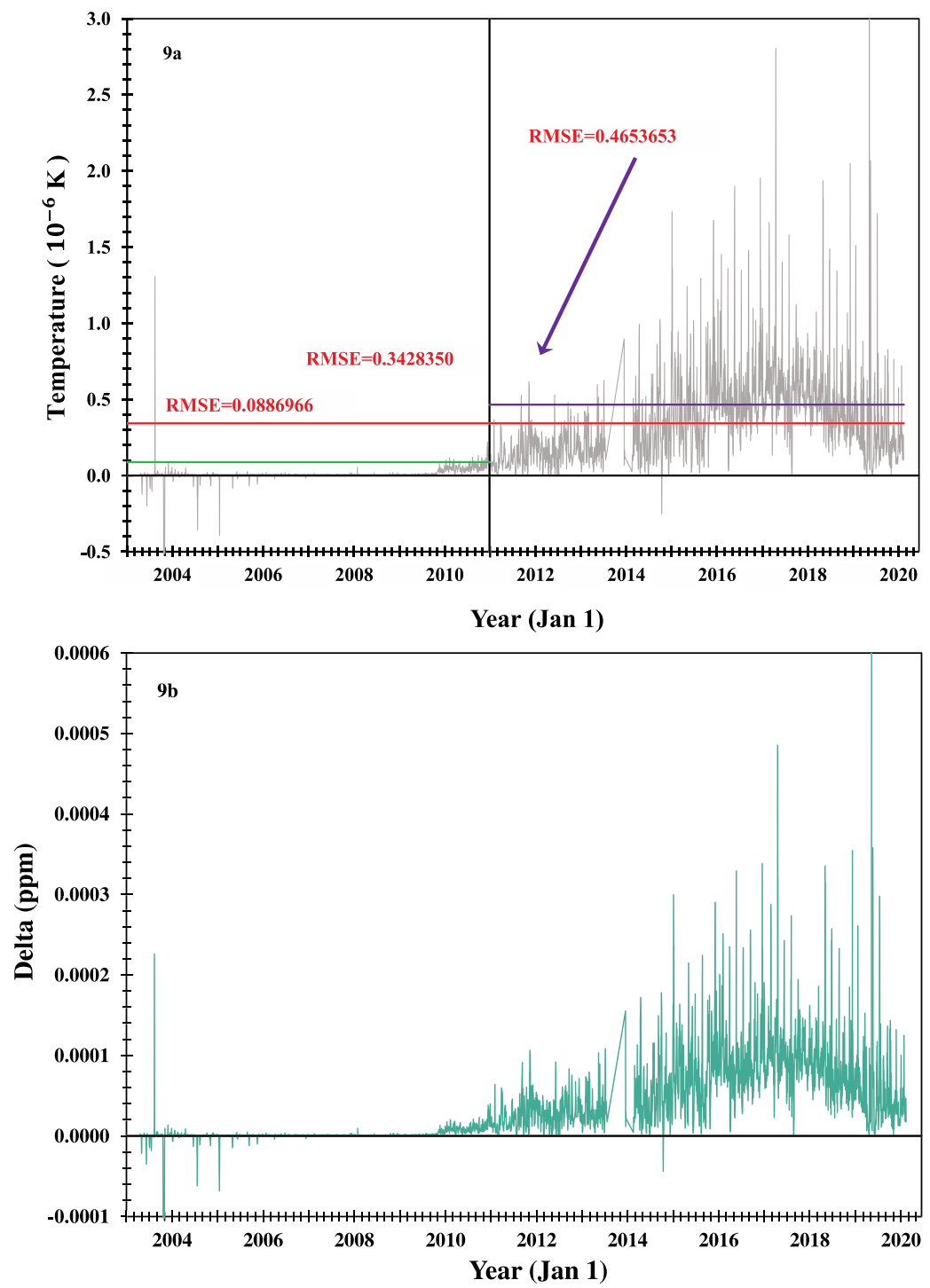

Figure 9 (a) The difference between the exact value of $T$ and the value obtained with the quadratic analytic approximation. (b) Delta quadratic approximation of $T$ using Equation 3.1 and expressed in parts per million (ppm). The positive values in this figure indicate that the quadratic analytic approximation underestimates the exact value of the temperature. Combining this result with that of Figure 7 shows that the exact value lies between the linear and quadratic analytic approximations.

Tables 1, 3 and 4 extend Table 2 to wavelengths $285.5 \mathrm{~nm}, 855.93 \mathrm{~nm}$, and $1547.09 \mathrm{~nm}$, respectively. As noted for $\mathrm{H} \alpha$, at these near-ultraviolet and near-infrared wavelengths, the linear fit model also has smaller RMSE than the linear analytic. Also, if we compare the two quadratic models, then again for $255.5 \mathrm{~nm}, 855.3 \mathrm{~nm}$, and $1547.09 \mathrm{~nm}$, the quadratic fit model wins, and for 255.5 and 855.3 it is by an even larger factor than it does for $\mathrm{H} \alpha$, by factors 10.34 and 7.78, respectively, while for $1547.09 \mathrm{~nm}$ the quadratic fit model wins over 

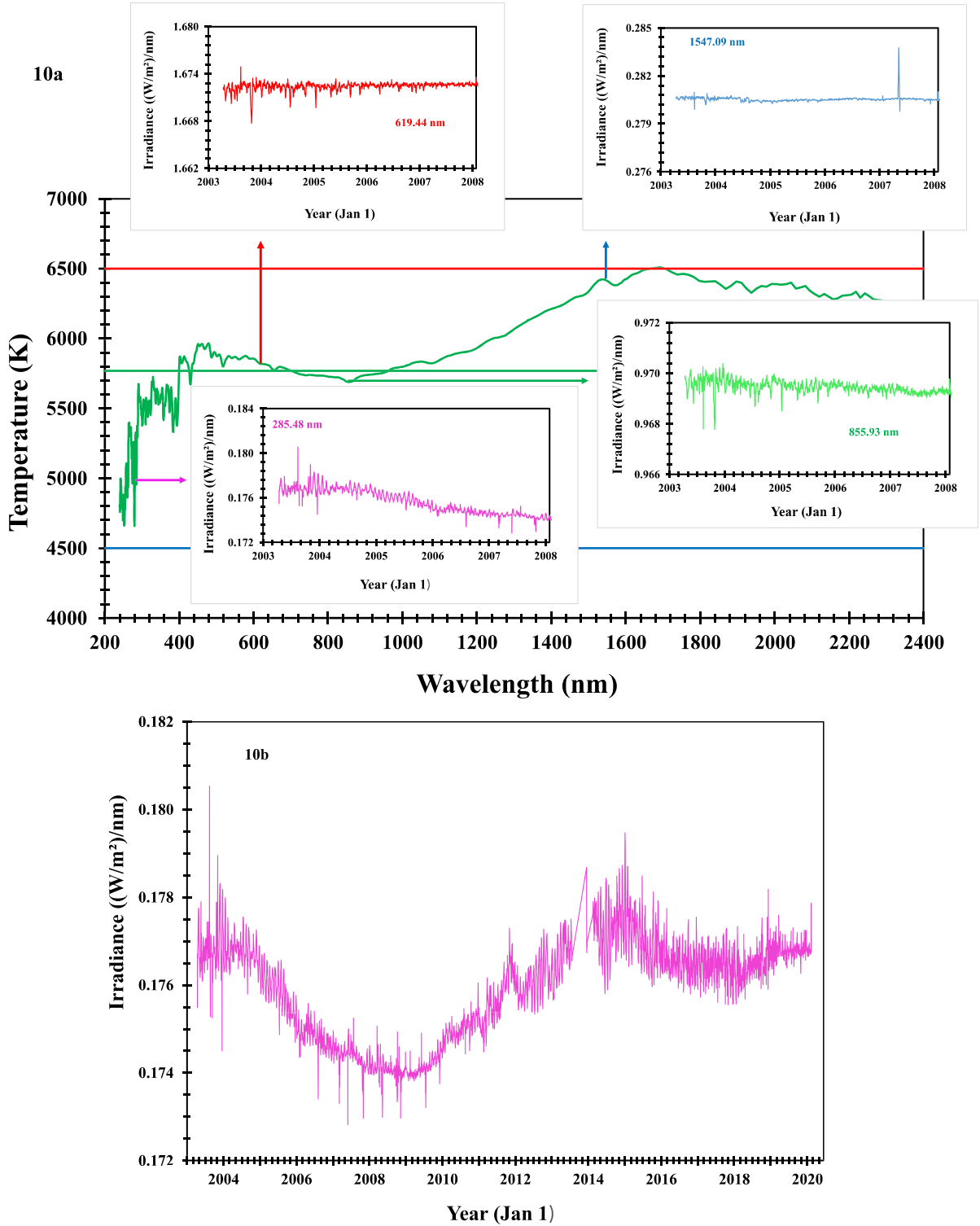

Figure 10 (a) Irradiance versus time during 2003-2008, for four chosen wavelengths, overlaid on a plot of temperature $T$ versus wavelength on the reference day, and indicating the reference-day temperatures at those same four chosen wavelengths. (b) Irradiance versus time during 2003-2020, for the shortest chosen wavelength, $285.48 \mathrm{~nm}$. (c) Irradiance versus time during 2003-2020, for the second chosen wavelength, $619.4 \mathrm{~nm}$. (d) Irradiance versus time during 2003-2020, for the third chosen wavelength, $855.93 \mathrm{~nm}$. (e) Irradiance versus time during $2003-2020$, for the longest chosen wavelength, $1547.09 \mathrm{~nm}$.

the quadratic analytic by a factor 2.00. If we take these four wavelengths as representative, then, the quadratic fit model is preferred, and nearly reproduces the exact values, despite the high precision of the quadratic analytic model. 

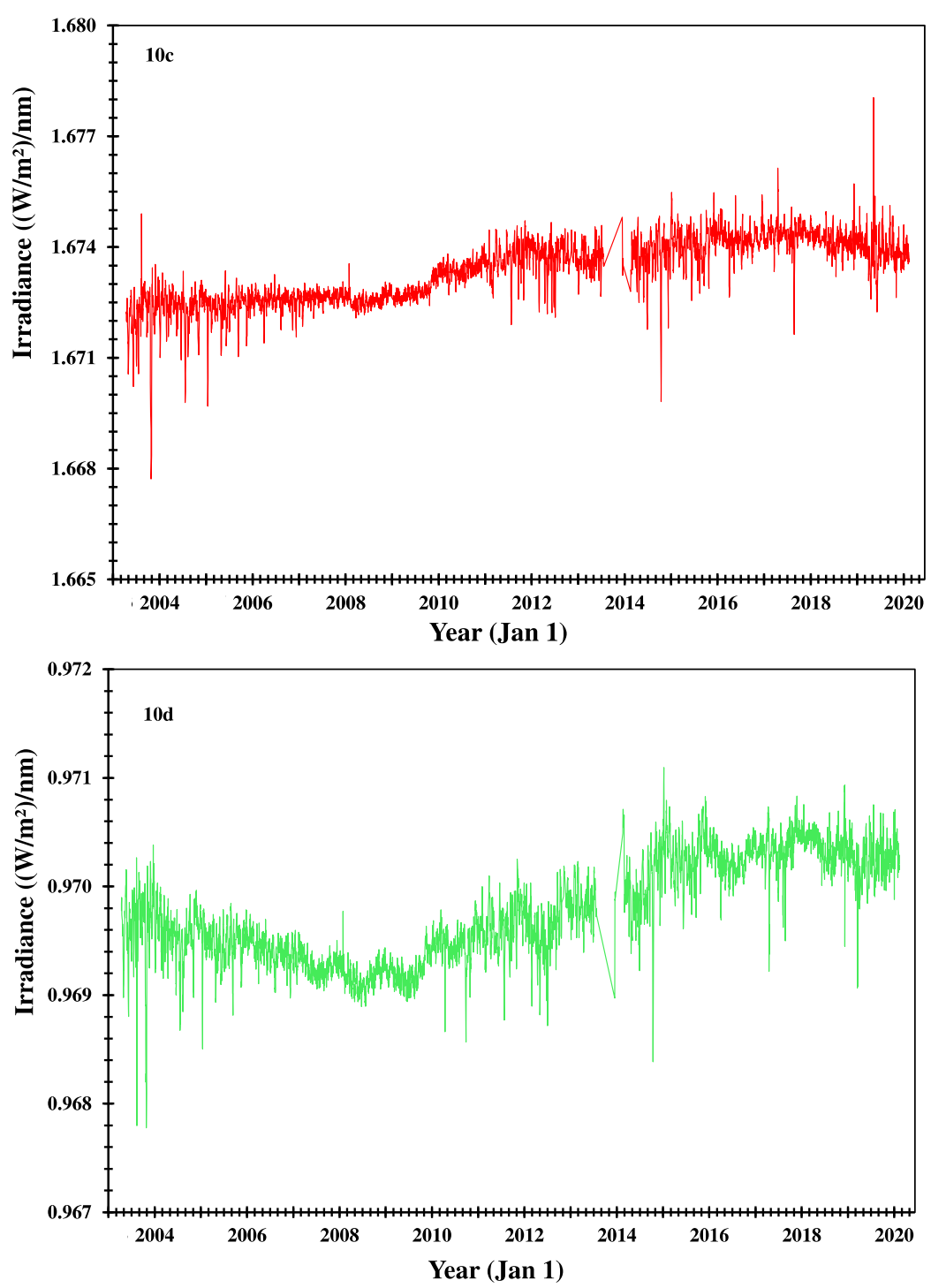

Figure 10 (Continued)

Some applications may not require such high precision. If we choose to restrict ourselves to linear models the fit model is still preferred, though it is a close call at $1547.09 \mathrm{~nm}$, where the linear analytic model RMSE is $1.05 \times$ larger than the linear fit, so has only a $5 \%$ improvement. At that wavelength, the linear analytic may be sufficient, and indeed an analytic approach has some advantages. For example, it may be optimized for a particular range of dates of particular interest, and the single coefficient interpreted as a "linear sensitivity" of temperature to irradiance at this wavelength.

Note that the SIM instrument registers a higher variability of spectral irradiance for shorter wavelengths, i.e., $285.5 \mathrm{~nm}$ and $355.93 \mathrm{~nm}$. This occurs because the more ener- 


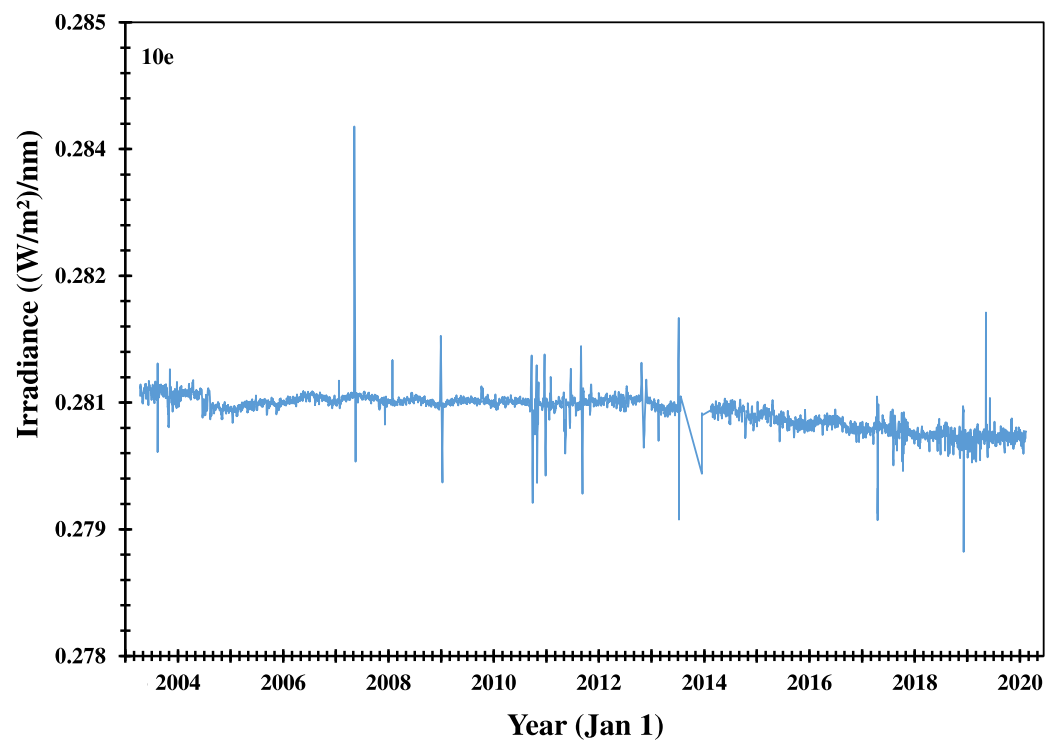

Figure 10 (Continued)

Table 1 Root-mean-square-error (RMSE) and the mean error (ME) of the temperature values obtained with the different models compared with exact temperature $(T)$ values, for 2003 to 2020 , and a wavelength of $285.48 \mathrm{~nm}$.

Table 2 RMSE and ME of the temperature values obtained with the different models compared with the exact temperature values, over the full range 2003 to 2020 , for a wavelength of $656.20 \mathrm{~nm}(\mathrm{H} \alpha)$.

\begin{tabular}{lll}
\hline Model & RMSE $\left[10^{-6} \mathrm{~K}\right]$ & Mean Error $\left[10^{-6} \mathrm{~K}\right]$ \\
\hline Linear analytic & $41,688.5145$ & $-31,954.3621$ \\
Quadratic analytic & 472.6288 & 313.800 \\
Linear fit & 8186.7338 & -0.0031 \\
Quadratic fit & 45.7226 & -0.0005 \\
\hline
\end{tabular}

\begin{tabular}{lll}
\hline Model & RMSE $\left[10^{-6} \mathrm{~K}\right]$ & Mean Error $\left[10^{-6} \mathrm{~K}\right]$ \\
\hline Linear analytic & 412.4455 & -277.1424 \\
Quadratic analytic & 0.3428 & 0.1945 \\
Linear fit & 144.9298 & 0.0144 \\
Quadratic fit & 0.1221 & 0.0304 \\
\hline
\end{tabular}

getic photons (according to the Planck-Einstein relationship $E=h c / \lambda$ ) allow for more transition and ionization processes than at near-infrared wavelengths, such as those shown in Figure 10, $855.3 \mathrm{~nm}$ and $1547.09 \mathrm{~nm}$.

Continuing with the plans for simplifying the calculations of the brightness temperature, which is the central objective of the article, Figure 11 shows the plots of the quotients of the linear analytic coefficients for certain wavelengths. Looking at the behavior of the curve of the quotients $\boldsymbol{a}^{\prime}$, a polynomial interpolation was obtained, as discussed in Appendix I. This provides a simple mathematical expression useful in calculating the linear coefficients for any wavelength in the range from $400 \mathrm{~nm}$ to $1800 \mathrm{~nm}$. With this, calculating the brightness temperature becomes simpler and faster than Equation E.18 with D.3, and valid for 
Table 3 RMSE and ME of the temperature values obtained with the different models compared with the exact temperature values from 2003 to 2020 for the wavelength $855.93 \mathrm{~nm}$

\begin{tabular}{lll}
\hline Model & RMSE $\left[10^{-6} \mathrm{~K}\right]$ & Mean Error $\left[10^{-6} \mathrm{~K}\right]$ \\
\hline Linear analytic & 423.5579 & -301.6674 \\
Quadratic analytic & 0.3408 & 0.2162 \\
Linear fit & 85.7852 & 0.0079 \\
Quadratic fit & 0.0438 & 0.0091 \\
\hline
\end{tabular}

Table 4 RMSE and ME of the temperature values obtained with the different models compared with the exact temperature values from 2003 to 2020 for the wavelength $1547.09 \mathrm{~nm}$.

\begin{tabular}{lll}
\hline Model & RMSE $\left[10^{-6} \mathrm{~K}\right]$ & Mean Error $\left[10^{-6} \mathrm{~K}\right]$ \\
\hline Linear analytic & 792.7592 & -213.7115 \\
Quadratic analytic & 4.5085 & -0.0978 \\
Linear fit & 758.1064 & 0.0087 \\
Quadratic fit & 2.2569 & 0.0108 \\
\hline
\end{tabular}

interpolating to wavelength bins not aligned with wavelength bins measured by the SIM instrument.

To compare the linear analytic and linear fit models, Figures 12 and 13 show the differences between the coefficients of the linear analytic approximation model, Equations E.10 and E.18 omitting the quadratic term, or G.5, and that of the linear fit model, Equation G.1. Note that the fit coefficient $\boldsymbol{a}$ in Equation G.1 is computed using $R$ software, and depends on the range of days supplied. This can range over the full set of days available from SORCE SIM (17 years of daily data). For comparison we also compute $a \mathrm{R} 1$ over the set of days in the first half of the data, that have the smaller or RSME values shown in Figure 7a, as well as $a \mathrm{R} 2$ over the late-day range, with larger RMSE. In short, early and late-year ranges are $\mathrm{R} 1=2003-2010$, and R2 $=2011-2020$. All three ranges, overall, R1 and R2 are shown in Figures 12 and 13. In the figures we can see that the values obtained with Equations G.1 and G.5 (with E.10) do not vary much for wavelengths less than $1400 \mathrm{~nm}$ and greater than $400 \mathrm{~nm}$, therefore the brightness temperature values that are calculated in that range of wavelengths also do not differ much, using the linear analytic and linear fit models. Note that $\boldsymbol{a} \mathrm{R} 1$ and $\boldsymbol{a} \mathrm{R} 2$ values lie on either side of the overall difference value of $\boldsymbol{a}$, which in every case lies in between, for each wavelength.

\section{Summary and Conclusions}

Our results and conclusions may be summarized as follows: (i) The linear and quadratic analytic approximation models, Equations E.18, with Equation E.10 for the linear term, and E.17 for the quadratic term, and E. 3 to compute $B$ from $S S I$, simplify calculations of solar brightness temperature $T$ on any chosen day for a fixed wavelength, with $B$ or SSI as a single variable. (ii) The linear analytic approximation overestimates the exact values of $T$, while the quadratic analytic approximation underestimates the exact values, but has much smaller RMSE (rms error) than the linear. (iii) By using the full dataset to find coefficients that minimize the RMSE we find linear and quadratic "fit" approximations that lie closer to the exact values for representative wavelengths, as can be seen by the "fit" RMSE values in Tables 1 to 4, being smaller than the corresponding analytic RMSEs, i.e., (fit RMSE)/(analytic RMSE) < 1 for both linear and quadratic cases, for near-ultraviolet, visible, and nearinfrared wavelengths. (iv) For wavelengths in between the tabulated ones, Equations I.1 and 

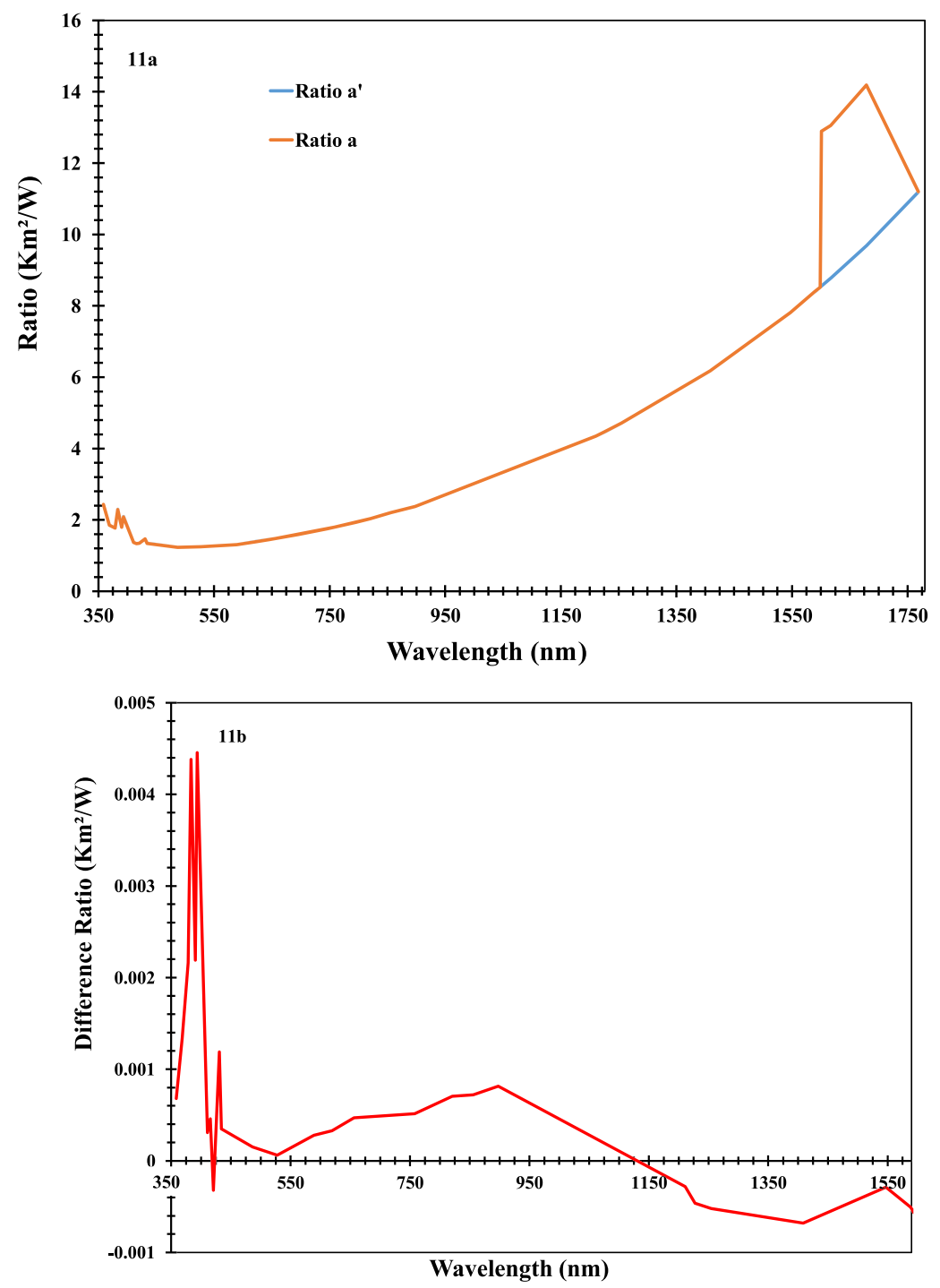

Figure 11 For each wavelength there is an associated linear "fit" coefficient, calculated using Mathematica and $R$ software. (a) The ratio obtained from the division of the linear coefficients between the respective wavelengths. (b) The differences between the ratios. (c) Comparison between the analytic ratio $\boldsymbol{a}^{\prime}$ and the value obtained with the polynomial interpolation in the same range of wavelengths, as in Equations I.1 and I.2.

I. 2 provide a smooth interpolating polynomial function of wavelength, which is simpler and faster to apply than Equation E.10 in the analytic case, or the $R$ software in the fit case, and accurate for any wavelength within a broad range across the peak of the SSI, extending into near-infrared wavelengths that are of particular importance in modeling Earth's climate.

The statistical measure used to understand the differences between values calculated by the linear and quadratic analytic approximation models with the exact values of $T$ obtained from Equation D.2 (or root finding in Mathematica software), is the RMSE (root-mean- 


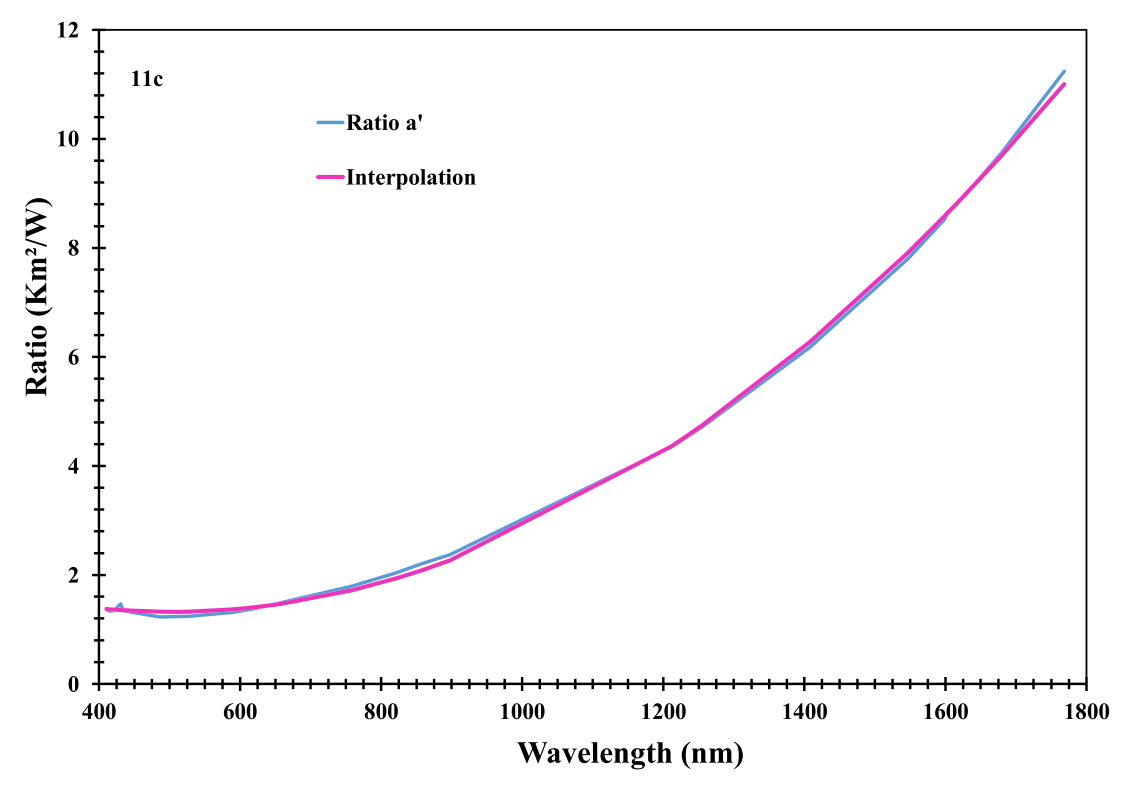

Figure 11 (Continued)

square-error). Figures 7a and 9a and Table 2 show that for the $\mathrm{H} \alpha$ wavelength the RMSE for the linear $\left(412.455 \times 10^{-6} \mathrm{~K}\right)$ and quadratic $\left(0.3428 \times 10^{-6} \mathrm{~K}\right)$ analytic approximation models are small, and therefore the deviations between the estimated and exact values are small. Table 1 shows that for a wavelength of $285.5 \mathrm{~nm}$ the RMSEs for both analytic models remain small, though larger than for $\mathrm{H} \alpha$. For both these wavelengths, the quadratic analytic model is superior to the linear analytic model. Tables 3 and 4 shows that for the longer nearinfrared wavelengths $855.93 \mathrm{~nm}$ and $1547.09 \mathrm{~nm}$ this pattern continues, with the quadratic analytic model being superior to the linear analytic. The fact that at all four wavelengths the quadratic analytic RMSE is smaller than the linear analytic RMSE suggests that further terms in the Taylor expansion may converge towards the exact over the full wavelength range. However, we do not have proof of convergence. Even if the series does converge, there is only a suggestion, not a guarantee, that it will converge to the exact value given by Equation D.2.

Comparisons of the linear analytic coefficient (Equation E.10 or G.5) with the coefficient of the linear least squares fit of the data performed with the statistical packages of $R$ software are shown in Figures 11 and 12. The linear fit model shows the line that best represents the entire data set, whereas the linear analytic approximation model has its maximum accuracy on the chosen reference day. Gaps in the data, the primary one being that which occurs from 2013-07-20 and 2014-03-12 (Harder, Beland, and Snow, 2019) have a direct influence on the coefficient of linear fit, because the solar spectrum measurement instruments SIM A and SIM B showed significant differences from the spectrum measured at the beginning of 2011, as can be seen for example in Figure 1 of the article of Harder, Beland, and Snow (2019).

Despite the good quality of the two analytic approximations, we find that the two fit models provide better "in between" approximations. The most accurate of the four approximations considered here is the quadratic fit model. We have seen that the brightness temperatures that it produces are in most cases indistinguishable from the exact temperatures that are found as roots of the equation that defines the brightness temperature, $S S I=\alpha_{s} B(T)$, 

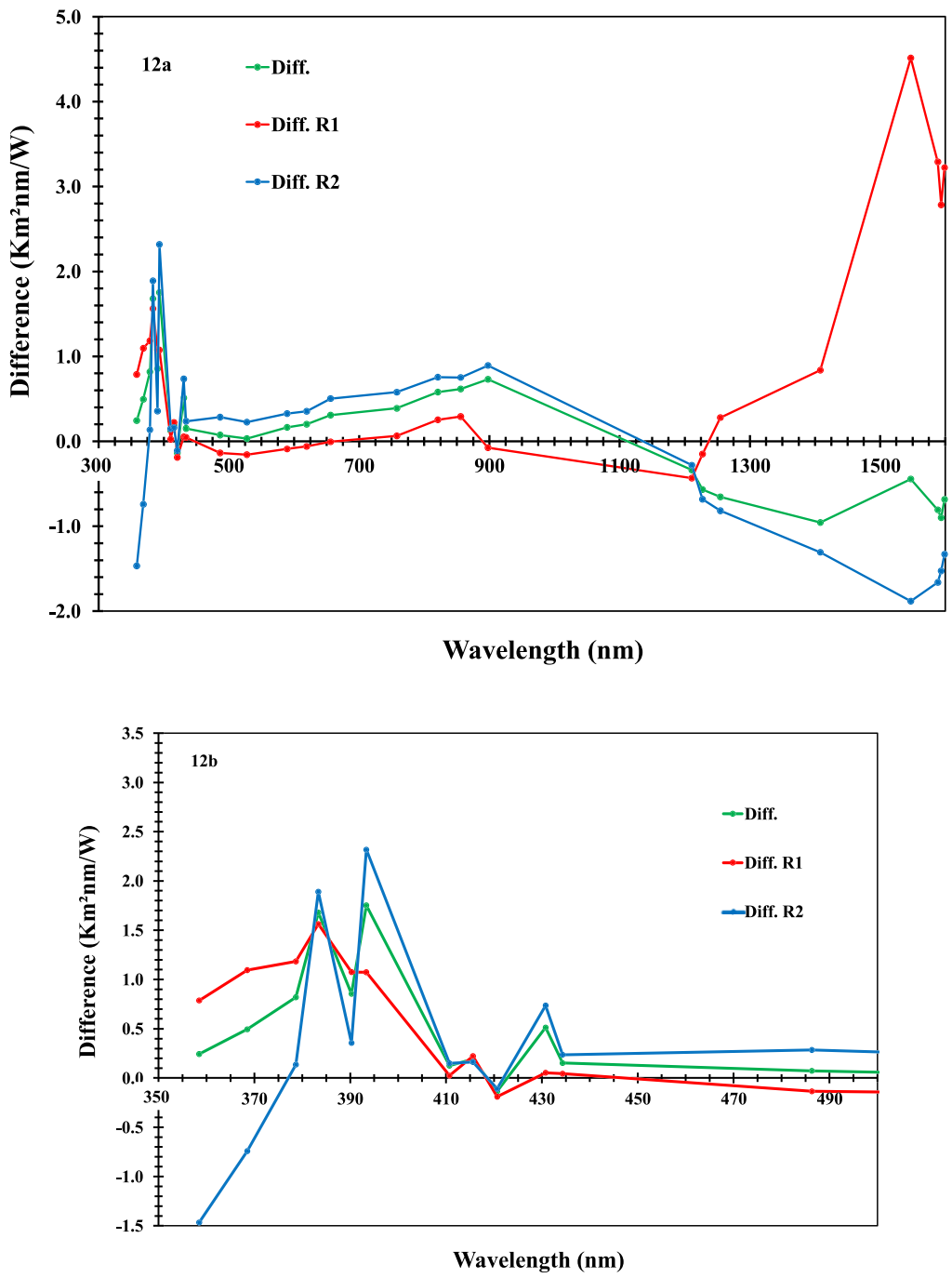

Figure 12 Three curves where: Diff is the difference between the analytic linear coefficient $\boldsymbol{a}^{\prime}$ obtained with Equations G.5 or E.10, and the fit linear coefficient $\boldsymbol{a}$ obtained with Equation G.1, for selected wavelengths, using either the complete data from 2003 to 2020 , or only early data in range R1 to compute $a \mathrm{R} 1$ or only late data in range $\mathrm{R} 2$ to compute $a \mathrm{R} 2$. In brief, R1 $=2003-2010$, while R2 $=2011-2020$. Note that $a \mathrm{R} 1$ and $\boldsymbol{a} \mathrm{R} 2$ values lie on either side of the overall difference value of $\boldsymbol{a}$, for each wavelength.

where $B$ is the Planck distribution, and $\alpha_{s}$ is the solid angle subtended by the Sun at the mean Earth distance.

There will soon be new opportunities to apply and extend this study. Both TIM and SIM instruments are now acquiring daily data onboard the International Space Station. The new record, begun on 2018-03-14, had sufficient overlap with SORCE to enable the prior dataset to be adjusted to match TSIS-1 (https://lasp.colorado.edu/lisird/data/sorce_sim_tav_ 13b/). Currently, TSIS-1 extends to 2021-07-20 and continues to be extended. TSIS-1 will be succeeded by TSIS-2, which is expected to continue the record beyond the peak of Solar 


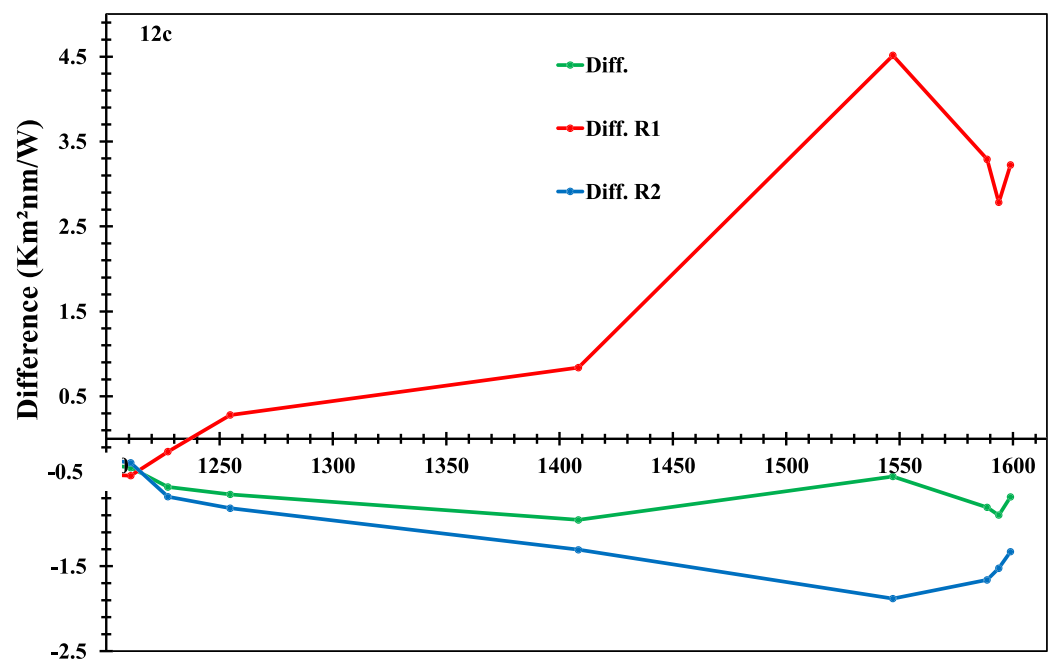

Wavelength (nm)

Figure 12 (Continued)

Cycle 25. We look forward to testing and applying the approximations studied here to future solar-cycle data, to enable improved understanding of the Sun's irradiance and temperature variations.

\section{Appendix A: Total Solar Irradiance and the Sun's Effective Temperature}

The Sun is not a blackbody, since the brightness temperature varies significantly with wavelength, as shown in Figure 1. However, we can define an "effective" radiative temperature $T_{\text {eff }}$, using the blackbody formula, with the Stefan-Boltzmann constant $\sigma=$ $5.670374 * 10^{-8} \mathrm{~W} / \mathrm{m}^{2} / \mathrm{K}^{4}$ as follows

$$
T S I=\alpha * \sigma T_{e f f}^{4}
$$

Here, TSI is the total solar irradiance (historically "solar constant"), while $\alpha$ is the ratio between the total area of the Sun with radius $R_{s}=6.957 * 10^{8} \mathrm{~m}$ divided by the area of a sphere centered on the Sun with radius equal to one astronomical unit $\mathrm{AU}=$ $149597870700.0 \mathrm{~m}$, so that

$$
\alpha=\frac{4 \pi R_{s}^{2}}{4 \pi(\mathrm{AU})^{2}}=\left(\frac{R_{s}}{\mathrm{AU}}\right)^{2}=2.16268 * 10^{-5} .
$$

The energy flow emitted by the Sun decreases as it diverges from the Sun's photosphere, becoming isotropically decreasing as $1 /$ distance $^{2}$. TSI and SSI values measured by satellites like SORCE are adjusted to the mean Earth-Sun distance of one AU, thus removing variations due to the satellite orbit. Earth receives a small fraction of the energy emitted by the Sun and recorded by satellites, and that fraction will be considered in the following. 

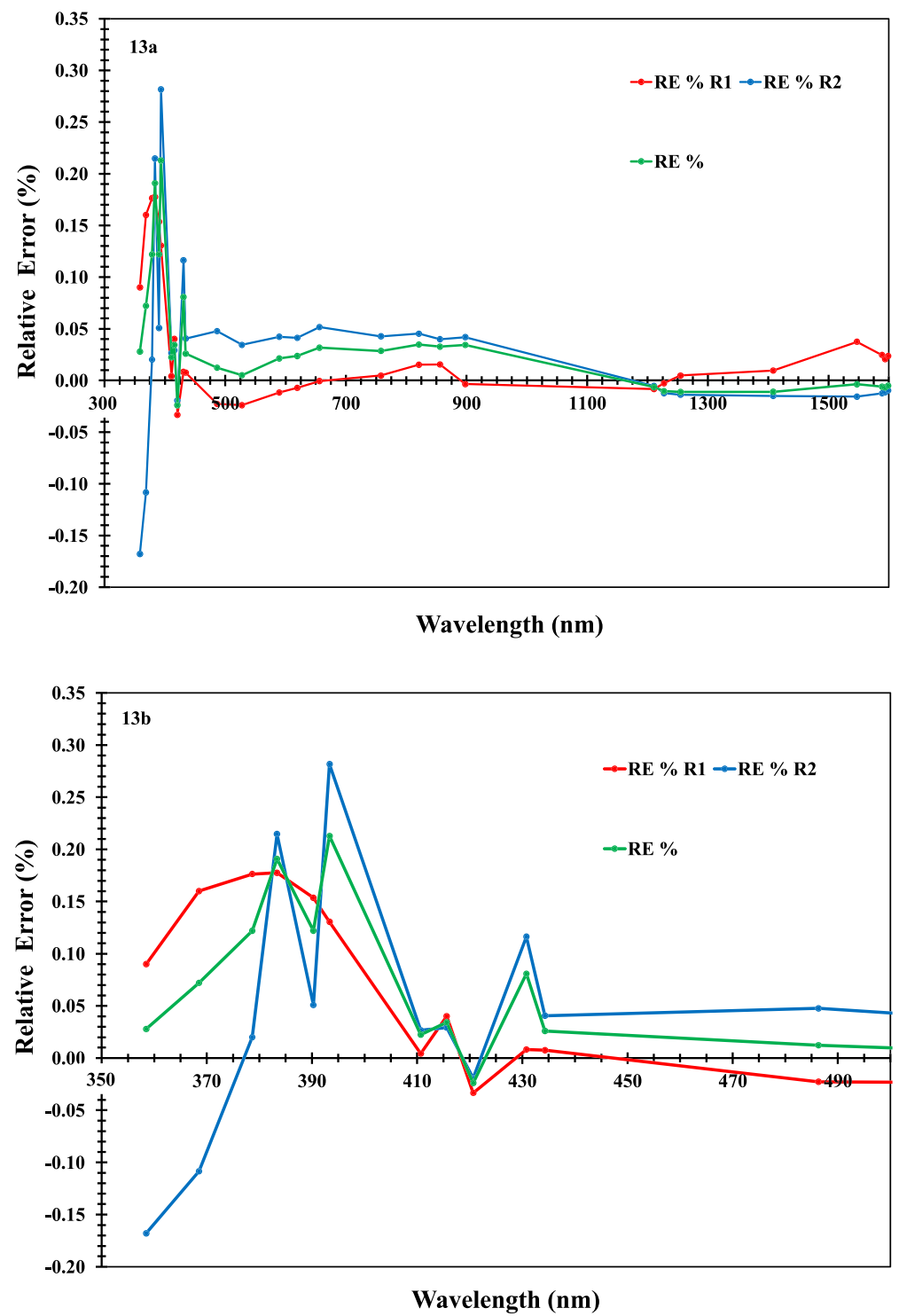

Figure 13 The relative error between the analytic linear coefficient $\boldsymbol{a}^{\prime}$ and the fit linear coefficient $\boldsymbol{a}$, where the fit coefficient $\boldsymbol{a}$ is calculated in the same three ways as in Figure 12, namely using the full available time period 2003-2020, or R1 = 2003-2010, or R2 = 2011 -2020 .

\section{Appendix B: Solar Luminosity and the Sun's Lifetime}

Questions of how the Sun shines, and how old it is, have been objects of interest since ancient times, but it was not until the scientific revolution that there was an opportunity to give definitive answers, first from classical physics, then using ideas from relativity, quantum mechanics and nuclear physics. With the development of modern theories, the answer became well understood (Bethe, 1939; Bahcall, 2000; Adelberger et al., 2011). 


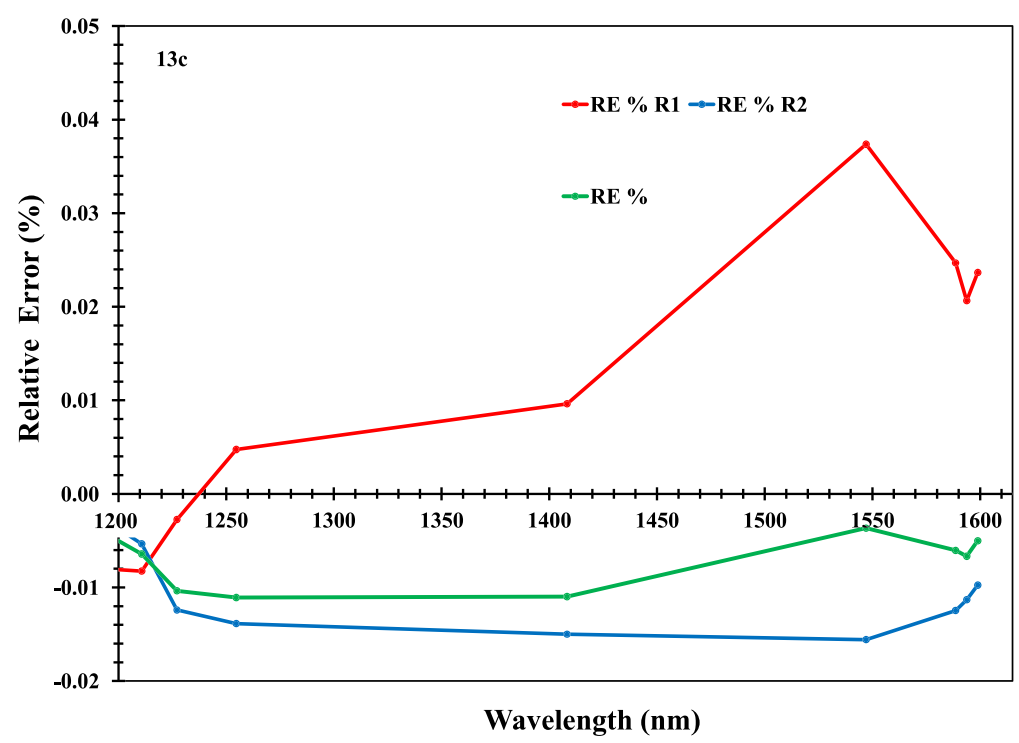

Figure 13 (Continued)

The solar luminosity, $L$, is the total solar power, the total radiative energy emitted from the Sun per second, isotropically in all directions. The best current estimate of $L$ relies on the measurements of TSI, which is the solar power per $\mathrm{m}^{2}$ at the mean Earth-Sun distance of one AU. To obtain $L$ from TSI, multiply by the total number of square meters on a sphere with radius equal to the Earth-Sun distance, so using the TIM value from Kopp and Lean, 2011 gives

$$
L=T S I *\left(4 \pi * \mathrm{AU}^{2}\right)=\left(1360.8 \frac{\mathrm{W}}{\mathrm{m}^{2}}\right) *\left(4 \pi * \mathrm{AU}^{2}\right)=3.82696 * 10^{26} \mathrm{~W} .
$$

The energy produced by nuclear reactions in the Sun's core is determined using Einstein's $E=m c^{2}$, where $\mathrm{m}$ is the mass loss in the primary reaction, which in the Sun is conversion of four $\mathrm{H}$ atoms into one He, as explained in 1939 by Hans Bethe in his classic 1939 paper "Energy production in stars," for which he won the Nobel Prize (Bethe, 1939).

Assuming the Sun's luminosity is approximately constant over the Sun's lifetime, then

$$
L * \text { Lifetime }=\text { Total Joules }=M_{s} c^{2} * 0.00723 * 0.1 /(31,556,952),
$$

where the Sun's mass $M_{s}=1.9885 \times 10^{30} \mathrm{~kg}$, the speed of light in vacuum is $c=$ $299,792,458 \mathrm{~m} / \mathrm{s}$, the mass loss fraction in the $4 \mathrm{H} \rightarrow \mathrm{He}$ process is 0.00723 , the core fraction in which nuclear reactions are self-sustaining is 0.1 , and the number of seconds per year is $31,556,952$. Dividing Equation B.2 by Equation B.1, the lifetime of the Sun can now be computed, with the result

$$
\text { Lifetime } \approx \frac{\text { Total Joules }}{L}=10.70 * 10^{9} \text { years. }
$$

If the constant value of $L$ is replaced by a linearly increasing $L$, while the Sun is also assumed to be about halfway through its lifetime, then the above estimate is not significantly altered, since a dimmer younger Sun is compensated by a brighter older Sun. 


\section{Appendix C: Earth's Temperature from TSI}

Earth intercepts a small fraction of the solar energy, casting a small shadow on the sphere of area $4 \pi * \mathrm{AU}^{2}$. That absorbed energy fraction is determined by the product of the TSI, the Earth's cross section $\left(\pi * R_{E}^{2}\right.$ ), and the Earth's absorptivity, or its albedo $\alpha=1$ - absorptivity. The absorbed fraction determines Earth's global mean temperature (North, Cahalan, and Coakley, 1981; Gray et al., 2010). Earth's temperature then determines the total thermal energy that Earth emits back into space. The balance between the absorbed solar energy, and the emitted thermal energy, determines Earth's effective radiative temperature, $T_{E}$. This condition of radiative equilibrium at the top of Earth's atmosphere is expressed as

$$
T S I *(1-\alpha) *\left(\pi * R_{E}^{2}\right)=\sigma T_{E}^{4} *\left(4 \pi * R_{E}^{2}\right) .
$$

Dividing through by Earth's surface area gives the global average energy emitted and absorbed in the form

$$
\sigma T_{E}^{4}=\left(\frac{T S I}{4}\right) *(1-\alpha)=\epsilon *(1-\alpha)
$$

where $\epsilon=T S I / 4$, and $\alpha=$ Earth albedo $=0.29$. (Note, the albedo symbol $\alpha$ used in Equation C.2 is not the $\alpha$ of Equation A.2.) Now, knowing that the energy absorbed and radiated by the Earth are equal, in thermal equilibrium, the effective temperature of the Earth can be calculated as

$$
T_{E}=\left(\frac{E_{a b s}}{\sigma}\right)^{0.25} .
$$

TSI impacts the average and long-term variability of Earth's temperature and, of course, its variations have impacted climate for millions of years (Kopp and Lean, 2011; Solanki, Krivova, and Haigh, 2013). TSI variations can be understood as a combined impact of variations in sunspots, and faculae, as well as variations occurring over the entire Sun. Models based on these have been key tools in studies of Earth's climate (e.g., Kopp and Lean, 2011; Foukal and Lean, 1985).

\section{Appendix D: “Exact" Solar Brightness Temperatures}

As already mentioned in previous sections, the Sun is not a pure blackbody. The SSI (solar spectral irradiance) has evident deviations from a pure Planck distribution, due to atomic absorption and ionization processes in the solar atmosphere. (See Figures 1 and 2.) An especially helpful way to study these deviations is by transforming SSI at each wavelength $\lambda$ into solar brightness temperature $T$. To do this, at each fixed wavelength, we solve the Planck distribution for $T$. That is, we solve

$$
B(\lambda, T)=\frac{k_{1}}{\lambda^{5} *\left(\exp \left(\frac{k_{2}}{\lambda T}\right)-1\right)},
$$

where $k_{1}=10^{20} c_{1}=1.19268 * 10^{20} \mathrm{~W} \mathrm{~m}^{2} / \mathrm{Sr}$ and $k_{2}=10^{7} c_{2}=1.43877 * 10^{7} \mathrm{Km}$ are constants, and the units of $B$ are $\mathrm{W} / \mathrm{m}^{2} / \mathrm{nm}$. Solving for $T$ gives the following, which we term the "exact" solar brightness temperature:

$$
T(\lambda, B)=\frac{k_{2}}{\lambda * \ln \left(\frac{k_{1}}{\lambda^{5} B}+1\right)} .
$$


To obtain the solar spectral irradiance SSI from the Planck distribution $B$ requires an integral over the solid angle of the Sun at the Earth's mean orbital distance. This gives

$$
S S I=\alpha_{S} B
$$

where the value of $\alpha_{s}=\pi * \alpha$, so from Equation A.2 we have $\alpha_{s}=6.79426 * 10^{-5}$. Note the wavelength $\lambda$ is kept fixed, and for each wavelength there is a corresponding brightness temperature $T$, determined by the value of temperature for which the satellite's SSI observation coincides with the Planck distribution for that $\lambda$ and $T$. Equivalently to Equation D.2, to solve Equation D.3 in Mathematica software, we use the initial condition $T=5770 \mathrm{~K}$ and $\alpha_{s}=\pi *\left(R_{s} / \mathrm{AU}\right)^{2}$, and apply the function FindRoot to Equation D.3, which gives the same values of $T$ as the explicit "exact" Equation D.2.

The next appendix shows how to approximately calculate the brightness temperatures $T$ for any fixed wavelength, having only the observed $S S I$ values (or equivalently $B$ ) as a variable, because all other parameters are defined on a single "reference day" so do not vary from day-to-day. It is important to remember that the wavelength is fixed, and consequently, the parameters $T_{o}, S S I_{o},(d T / d S S I)_{o}$ and higher derivatives (evaluated on the reference day) vary with wavelength. For the SIM data used in this article to produce the plots, the wavelengths range from $240 \mathrm{~nm}$ to $2416 \mathrm{~nm}$. For each wavelength in this range, there is a set of parameters that can be used to determine a time series of brightness temperatures $T$ for all other days in the date range [2003-04-14 to 2020-02-26].

\section{Appendix E: Analytic Approximations for Brightness Temperature}

This appendix derives two simple analytic representations of the daily brightness temperatures that take advantage of the fact that, at a given wavelength, the SSI values are very nearly equal from day-to-day, and typically vary by less than $1 \%$. The analytic approximations express the daily temperature values on any given day, $T$, at each fixed wavelength, by a Taylor expansion of the exact value of $T$ as an analytic function of $S S I$, as given in Equation D.2. The expansion is about the value of SSI and $T$ on a given "reference" day $\left(T_{o}, S S I_{o}\right)$, as follows

$$
T=T_{0}+\left(\frac{d T}{d S S I}\right)_{o}\left(S S I-S S I_{o}\right)+\frac{1}{2}\left(\frac{d^{2} T}{d S S I^{2}}\right)_{o}\left(S S I-S S I_{o}\right)^{2}+\cdots
$$

We focus on the "linear approximation" that keeps just the first derivative, and then the "quadratic approximation" that keeps the first two derivatives. Higher-order terms will be neglected, except in the discussion of convergence. Since SSI is directly proportional to $B$ by a constant rescaling, as given in Equation D.3, we may write E.1 as

$$
T=T_{o}+\left(\frac{d T}{d B}\right)_{o}\left(B-B_{o}\right)+\frac{1}{2}\left(\frac{d^{2} T}{d B^{2}}\right)_{o}\left(B-B_{o}\right)^{2}+\cdots
$$

In order to compute the first and second derivatives via the chain rule, we introduce two new variables, $y$ and $z$, as follows: Let

$$
y=\ln (z)=\ln \left[\frac{k_{1}}{\lambda^{5} B}+1\right]
$$


so that

$$
z=e^{y}
$$

and

$$
z-1=\frac{k_{1}}{\lambda^{5} B} .
$$

Then,

$$
\frac{d y}{d z}=\frac{1}{z}=\frac{1}{e^{y}}
$$

and

$$
\frac{d z}{d B}=\frac{-k_{1}}{\lambda^{5} B^{2}}=\frac{-\lambda^{5}}{k_{1}} *\left(e^{y}-1\right)^{2} .
$$

Therefore,

$$
\frac{d y}{d z} * \frac{d z}{d B}=\frac{-\lambda^{5}}{k_{1}} * \frac{\left(e^{y}-1\right)^{2}}{e^{y}} .
$$

Equations D.2 and E.3 imply

$$
T=\frac{k_{2}}{\lambda y}=T(y(z(B))) .
$$

We may compute the derivative of Equation E.9 using the chain rule, employing Equation E.8, to obtain

$$
\frac{d T}{d B} \equiv T^{(1)}=\frac{d T}{d y} *\left[\frac{d y}{d z} * \frac{d z}{d B}\right]=\frac{-k_{2}}{\lambda y^{2}} *\left[\frac{-\lambda^{5}}{k_{1}} * \frac{\left(e^{y}-1\right)^{2}}{e^{y}}\right]=\frac{k_{2} \lambda^{4}}{k_{1} y^{2}} *\left[\frac{\left(e^{y}-1\right)^{2}}{e^{y}}\right] .
$$

To evaluate E. 10 on the reference day, we set $B=B_{o}$ equal to the value on that day, compute $y=y_{o}$ from Equation E.3, and substitute that into Equation E.10. In order to compute the second derivative, we note that Equation E.10 is already in the form analogous to E.9, namely

$$
T^{(1)}=T^{(1)}(y(z(B))) .
$$

Therefore, as in computing Equation E.10, we take the derivative of E.11 using the chain rule, E. 8 and E.10 to obtain

$$
\begin{aligned}
T^{(2)} & =\frac{d T^{(1)}}{d y} *\left[\frac{d y}{d z} * \frac{d z}{d B}\right] \\
& =\frac{k_{2} \lambda^{4}}{k_{1}} *\left[\frac{d}{d y}\left(\frac{1}{y^{2}}\right) * \frac{\left(e^{y}-1\right)^{2}}{e^{y}}+\frac{1}{y^{2}} \frac{d}{d y}\left(\frac{\left(e^{y}-1\right)^{2}}{e^{y}}\right)\right] *\left[\frac{-\lambda^{5}}{k_{1}} * \frac{\left(e^{y}-1\right)^{2}}{e^{y}}\right] .
\end{aligned}
$$


On the right side we applied the product rule to compute $d T^{(1)} / d y$ from Equation E.10, giving the two terms in the left square brackets, and used Equation E.8 to substitute into the right square brackets. Evaluating the first term in the left bracket of Equation E.12 allows us to factor out $1 / y^{2}$ from both terms. We also combine the rightmost constant $-\lambda^{5} / k_{1}$ with the leftmost constant $k_{2} \lambda^{4} / k_{1}$ to yield the following

$$
T^{(2)}=\frac{-k_{2} \lambda^{9}}{k_{1}^{2}} *\left(\frac{1}{y^{2}}\right) *\left[\left(\frac{-2}{y}\right) * \frac{\left(e^{y}-1\right)^{2}}{e^{y}}+\frac{d}{d z}\left(\frac{(z-1)^{2}}{z}\right) \frac{d z}{d y}\right] *\left[\frac{\left(e^{y}-1\right)^{2}}{e^{y}}\right] .
$$

We apply the product rule to the remaining derivative in the second term in the left-hand brackets, and use Equation E.4, which implies $d z / d y=z$, to give

$T^{(2)}=\frac{-k_{2} \lambda^{9}}{k_{1}^{2}} *\left(\frac{1}{y^{2}}\right) *\left[\left(\frac{-2}{y}\right) * \frac{\left(e^{y}-1\right)^{2}}{e^{y}}+\left(\frac{2(z-1)}{z}-\frac{(z-1)^{2}}{z^{2}}\right) * z\right] *\left[\frac{\left(e^{y}-1\right)^{2}}{e^{y}}\right]$.

In the second term within the left square brackets, we distribute the $z$, then factor out $\frac{(z-1)^{2}}{z}$ to yield

$$
T^{(2)}=\frac{-k_{2} \lambda^{9}}{k_{1}^{2}} *\left(\frac{1}{y^{2}}\right) *\left[\left(\frac{-2}{y}\right) * \frac{\left(e^{y}-1\right)^{2}}{e^{y}}+\left(\frac{2 z}{z-1}-1\right) * \frac{(z-1)^{2}}{z}\right] *\left[\frac{\left(e^{y}-1\right)^{2}}{e^{y}}\right] .
$$

We substitute $\frac{(z-1)^{2}}{z}=\frac{\left(e^{y}-1\right)^{2}}{e^{y}}$ and factor that from both bracketed terms in Equation E.15, and combine the remaining terms with their common denominator to give

$$
T^{(2)}=\frac{-k_{2} \lambda^{9}}{k_{1}^{2}} *\left(\frac{1}{y^{2}}\right) *\left[\frac{-2(z-1)+y(z+1)}{y(z-1)}\right] *\left[\left(\frac{\left(e^{y}-1\right)^{2}}{e^{y}}\right)^{2}\right] .
$$

Finally, using $z=e^{y}$ and simplifying gives the final result

$$
T^{(2)}=\frac{-k_{2} \lambda^{9}}{k_{1}^{2}} *\left[2+y+e^{y}(y-2)\right] *\left[\left(\frac{e^{y}-1}{y}\right)^{3} / e^{2 y}\right] .
$$

To evaluate Equation E.17 on the reference day, just as for Equation E.10, we set $B=B_{o}$, equal to the value observed on that day, substitute that into Equation E. 3 to compute $y=y_{o}$, and substitute the value of $y=y_{o}$ into Equation E.17. Substituting these first and second derivatives of $T$ evaluated on the reference day into Equation E.2, and neglecting all higherorder terms, we obtain the quadratic analytic approximation given by

$$
T=T_{o}+\left(\frac{d T}{d B}\right)_{o}\left(B-B_{o}\right)+\frac{1}{2}\left(\frac{d^{2} T}{d B^{2}}\right)_{o}\left(B-B_{o}\right)^{2},
$$

where the linear term is computed using E.10, and the quadratic term is computed using E.17. Omitting the quadratic term in E.18 gives the linear analytic approximation.

\section{Appendix F: The Sun's Effective Temperature}

Here, we derive a linear approximation for the "effective" temperature $T_{e f f}$, Equation F.4, associated with the total solar irradiance, TSI. This is a simpler case than for SSI, since 
for TSI, the Stefan-Boltzmann equation makes the exact $T_{\text {eff }}$ a simple analytic function of TSI, given in Equation F.1. As mentioned before, the Sun is not a blackbody, but we can calculate its associated effective temperature by using the Stefan-Boltzmann Equation and using TSI (total solar irradiance) measured directly by satellites above the atmosphere, by solving Equations A.1 and A.2 to obtain

$$
T_{e f f}=\left(\frac{T S I}{\alpha \sigma}\right)^{0.25},
$$

where $\sigma=5.670374 * 10^{-8} \mathrm{~W} / \mathrm{m}^{2} / \mathrm{K}^{4}$ is the Stefan-Boltzmann constant, and from Equation A. $2 \alpha=2.16268 * 10^{-5}$. Taking the derivative of F.1 gives an expression for the change in effective temperature with a change in TSI as follows:

$$
\frac{d T_{\text {eff }}}{d T S I}=\frac{d}{d T S I}\left(\left(\frac{T S I}{\alpha \sigma}\right)\right)^{0.25} .
$$

Simplifying F.2 and evaluating on the reference day gives

$$
\left(\frac{d T_{e f f}}{d T S I}\right)_{o}=\frac{1}{4} *\left(\frac{T S I_{o}}{\alpha \sigma}\right)^{0.25} * \frac{1}{T S I_{o}}=\frac{1}{4} * \frac{\left(T_{e f f}\right)_{o}}{T S I_{o}} .
$$

Substituting into Equation F.3 the values on the reference day, 2008-08-24, which are $\left(T S I_{o},\left(T_{\text {eff }}\right)_{o}\right)=\left(1360.4704 \mathrm{~W} / \mathrm{m}^{2}, 5771.2685 \mathrm{~K}\right)$, we obtain

$$
\left(\frac{d T_{e f f}}{d T S I}\right)_{\mathrm{o}}=1.06053 \frac{\mathrm{K}}{\mathrm{W} / \mathrm{m}^{2}} .
$$

Since TSI typically varies by about $0.1 \%$ or less, Equation F.4 is quite accurate for most days. An extreme case is the "Halloween" event of 2003-10-29, when a large sunspot grouping dropped the temperature by about $3.6670 \mathrm{~K}$ below the reference day $T_{\text {eff }}$ on 2008-08-24. Equation F.4 estimates a $3.6605 \mathrm{~K}$ decrease from the reference day, i.e., a $0.0065 \mathrm{~K}$ underestimate, which is $0.1773 \%$ of the drop, or $0.0001 \%$ of $\left(T_{e f f}\right)_{o}=5771.2685 \mathrm{~K}$. Substituting the 2003 "Halloween" values of $T_{\text {eff }}$ and TSI into Equation F.3 gives instead of F.4 the coefficient 1.06255. This day of minimum $T_{\text {eff }}$ is also the day of maximum coefficient of sensitivity over the full 17-year SORCE SIM record and is $19 \%$ larger than the coefficient on the reference day, shown in Equation F.4.

Conversely, the minimum coefficient over the 17 years occurs on the day of maximum $T_{\text {eff }}$, which is $5773.1820 \mathrm{~K}$, which occurred on 2015-02-26. That minimum coefficient is $1.05947,10 \%$ less than the reference-day ratio in F.4. The average coefficient over all days is 1.06029 . The fact that this average value is $2 \%$ less than the reference day's implies that the linear approximation in Equation F.4 typically slightly overestimates the changes in $T_{\text {eff }}$. The same is true for SSI: the linear analytic approximation of brightness temperature $T$, obtained by dropping the quadratic term in Equation E.18, also has a positive mean error, or bias, as shown for four representative wavelengths in Tables $1-4$. Those tables also show that inclusion of the quadratic term in E.18 largely removes this positive bias, leaving a very small mean error, and small RMSE, as discussed in more detail in the text.

In principle, there are two ways to determine the total solar irradiance (TSI). The first is by using the SORCE TIM instrument to obtain a direct measurement. The second is to use the SSI measured by the SIM instrument and integrate as wide a range of wavelengths as possible. As expected, there is a shortfall in the value computed by integrating the 
SIM data compared to what is measured by TIM, mainly due to missing energy above the longest wavelengths measured by SIM, approximately $2400 \mathrm{~nm}$. This TIM-SIM difference is shown in Figure 2 of the article by Harder, Beland, and Snow (2019), and amounts to $146.128 \mathrm{~W} / \mathrm{m}^{2}$. This must be subtracted from the value measured by TIM, or added to the integrated SIM value, for comparisons to be made between TIM and SIM. In this paper we focus on SSI, though both SSI and TSI must be considered in the study of Earth's climatic variations.

\section{Appendix G: Linear and Quadratic Fit Model}

Comparing Figures 7 and 9 shows that the linear analytic approximation, using only the first two terms in E.18, overestimates the exact $T$, given in D.2, while the quadratic approximation, using all three terms in E.18, though closer to the exact, slightly underestimates. To consider a possible "in between" approximation, this appendix introduces linear and quadratic "fit" models. These statistical "fit" models calculate the brightness temperature as a function of wavelength using $R$ software. In the linear and quadratic analytic approximation models discussed in earlier appendices, estimates of solar brightness temperatures $T$ are made based on the measured solar spectrum of the chosen reference day, and the exact brightness temperatures computed for that day, which occurs during a time of minimum solar activity. By contrast, the fit models we discuss below take into consideration the statistical properties of the full set of daily data over the 17 years of the SORCE mission.

A statistical model that provides a least square fit to the solar spectral irradiance (SSI) data obtained in the $R$ software with linear regression may be written as

$$
T=a * S S I+b,
$$

where $a, b$ are constants for a specific wavelength and $T$ is the brightness temperature. In the same way, $R$ software may compute a least squares quadratic fit of the form

$$
T=C * S S I^{2}+A * S S I+B .
$$

These two fit models express linear and quadratic dependences, respectively, between $S S I$ and $T$. We obtain, using code developed in open-source $R$ software, simple models that best fit the data, for which the mean square error is minimized.

We rewrite the analytic Equation E.1 (or the equivalent E.2), up to the linear term, as follows:

$$
\begin{gathered}
T=T_{o}+\left(\frac{d T}{d S S I}\right)_{o} * S S I-\left(\frac{d T}{d S S I}\right)_{o} * S S I_{o} \\
T=\left(\frac{d T}{d S S I}\right)_{o} * S S I+T_{o}-\left(\frac{d T}{d S S I}\right)_{o} * S S I_{o} \\
T=a^{\prime} * S S I+b^{\prime}
\end{gathered}
$$

which has a similar form to Equation G.1. This will allow us to make a comparison between values of the constants that appear in Tables 7 and 9. The linear analytic coefficient, which appears in Table 6, and $S S I_{o}$ are constant, because they are evaluated for the data of the reference day that appears in Table 5. It is important to note that the constant $a^{\prime}$ defined in this part is the same constant given in the linear analytic term in Equation E.1. 
Table 5 In order to approximate the values of the temperature $T$, for a chosen set of wavelengths, on any chosen date, using Equation E.18, the values of $T$ and $S S I$ on the reference day $\left(T_{o}, S S I_{o}\right)$ are needed, as are the values of SSI on the day that the approximate values of $T$ are desired, for instance 2011-10-10. Also displayed in this table are the "exact" temperature values calculated by Equation D.2, and verified by solving Equation D.3 by root finding in Wolfram Mathematica for each wavelength, on the date of our example, 2011-10-10.

\begin{tabular}{lllll}
\hline $\begin{array}{l}\text { Wavelength } \\
{[\mathrm{nm}]}\end{array}$ & $\begin{array}{l}S S I_{o} \\
{\left[\frac{\mathrm{W}}{\mathrm{m}^{2}} / \mathrm{nm}\right]}\end{array}$ & $\begin{array}{l}T_{o} \\
{[\mathrm{~K}]}\end{array}$ & $\begin{array}{l}S S I \\
{\left[\frac{\mathrm{W}}{\mathrm{m}^{2}} / \mathrm{nm}\right]}\end{array}$ & $\begin{array}{l}\text { Exact value of } \\
T[\mathrm{~K}]\end{array}$ \\
\hline 285.48 & 0.1739754 & 4985.44659842 & 0.1759321 & 4990.9681473 \\
656.20 & 1.526558 & 5772.41067100 & 1.527622 & 5773.4459772 \\
855.93 & 0.9690168 & 5688.34171545 & 0.9696425 & 5689.5197810 \\
1547.09 & 0.2805222 & 6417.67574425 & 0.2805273 & 6417.7373565 \\
\hline
\end{tabular}

Table 6 Values of linear $\left(\mathrm{K} /\left(\left(\mathrm{W} / \mathrm{m}^{2}\right) / \mathrm{nm}\right)\right)$ and quadratic $\left(\mathrm{K} /\left(\left(\mathrm{W} / \mathrm{m}^{2}\right) / \mathrm{nm}\right)^{2}\right)$ coefficients are shown, as given in Equations E.10 and E.17, evaluated for the data of the reference day 2008-08-24, taken from Table 5 .

\begin{tabular}{lcc}
\hline $\begin{array}{l}\text { Wavelength } \\
{[\mathrm{nm}]}\end{array}$ & $\begin{array}{l}\text { Quadratic coefficient } \\
\left(\frac{d^{2} T}{d S S I^{2}}\right)_{o}\end{array}$ & $\begin{array}{l}\text { Linear coefficient } \\
\left(\frac{d T}{d S S I}\right)_{o}\end{array}$ \\
\hline 285.48 & -13070.296 & 2834.568 \\
656.20 & -323.644 & 973.204 \\
855.93 & -797.737 & 1883.046 \\
1547.09 & -7693.756 & 12080.859 \\
\hline
\end{tabular}

Table 7 Temperature values on 2011-10-10 for four wavelengths using the linear approximation of Equation G.5, compared with the exact value obtained using Equation D.2, and also (as a check) by solving Equation D.3 in Wolfram Mathematica.

\begin{tabular}{llllr}
\hline $\begin{array}{l}\text { Wavelength } \\
{[\mathrm{nm}]}\end{array}$ & $\begin{array}{l}\text { Constant } a^{\prime} \\
{\left[\mathrm{K} /\left(\frac{\mathrm{W}}{\mathrm{m}^{2}} / \mathrm{nm}\right)\right]}\end{array}$ & $\begin{array}{l}\text { Constant } b^{\prime} \\
{[\mathrm{K}]}\end{array}$ & $\begin{array}{l}\text { Estimated } \\
T[\mathrm{~K}]\end{array}$ & $\begin{array}{l}\text { Difference } \\
{[\mathrm{K}]}\end{array}$ \\
\hline 285.48 & 2834.568 & 4492.301 & 4990.9929982 & $-24,851.0$ \\
656.20 & 973.204 & 4286.758 & 5773.4461603 & -183.1 \\
855.93 & 1883.046 & 3863.639 & 5689.5199371 & -156.1 \\
1547.09 & 12080.859 & 3028.727 & 6417.7373566 & -0.1 \\
\hline
\end{tabular}

We follow a similar procedure for the quadratic analytic model, rewriting Equation E.1 to obtain,

$$
T=C^{\prime} * S S I^{2}+A^{\prime} * S S I+B^{\prime},
$$

which is a mathematical expression similar to Equation G.2, where the values of the constants are given as:

$$
\begin{gathered}
C^{\prime}=\frac{1}{2}\left(\frac{d^{2} T}{d S S I^{2}}\right)_{o} \\
A^{\prime}=\left(\frac{d T}{d S S I}\right)_{o}-\left(\frac{d^{2} T}{d S S I^{2}}\right)_{o} * S S I_{o},
\end{gathered}
$$


Table 8 Temperature values of 2011-10-10 for four wavelengths using the quadratic approximation model of Equation G.6, compared with the exact value obtained using Equation D.2, and checked by solving Equation D.3 in Wolfram Mathematica.

\begin{tabular}{llllll}
\hline $\begin{array}{l}\text { Wavelength } \\
{[\mathrm{nm}]}\end{array}$ & $\begin{array}{l}\text { Constant } C^{\prime} \\
{\left[\mathrm{K} /\left(\mathrm{W} / \mathrm{m}^{2} / \mathrm{nm}\right)^{2}\right]}\end{array}$ & $\begin{array}{l}\text { Constant } A^{\prime} \\
{\left[\mathrm{K} /\left(\mathrm{W} / \mathrm{m}^{2} / \mathrm{nm}\right)\right]}\end{array}$ & $\begin{array}{l}\text { Constant } B^{\prime} \\
{[\mathrm{K}]}\end{array}$ & $\begin{array}{l}\text { Estimated } T \\
{[\mathrm{~K}]}\end{array}$ & $\begin{array}{l}\text { Difference } \\
{[\mathrm{K}]}\end{array}$ \\
\hline 285.48 & -6535.148147 & 5108.478341 & 4294.499239 & 4990.9679773 & 0.00016998 \\
656.20 & -161.8219945 & 1467.265593 & 3909.651272 & 5773.4459771 & 0.00000008 \\
855.93 & -398.8687194 & 2656.066599 & 3489.103717 & 5689.5197809 & 0.00000007 \\
1547.09 & -3846.878 & 14239.12872 & 2726.005277 & 6417.7373565 & 0.00000000 \\
\hline
\end{tabular}

Table 9 Parameters "a" and "b" of the linear fit model (G.1) for each wavelength, which were obtained with $R$ software, necessary to estimate the temperature on 2011-10-10.

\begin{tabular}{lllll}
\hline $\begin{array}{l}\text { Wavelength } \\
{[\mathrm{nm}]}\end{array}$ & $\begin{array}{l}\text { Constant } a \\
{\left[\mathrm{~K} /\left(\mathrm{W} / \mathrm{m}^{2} / \mathrm{nm}\right)\right]}\end{array}$ & $\begin{array}{l}\text { Constant } b \\
{[\mathrm{~K}]}\end{array}$ & $\begin{array}{l}\text { Estimated } T \\
{[\mathrm{~K}]}\end{array}$ & $\begin{array}{l}\text { Difference } \\
{[\mathrm{K}]}\end{array}$ \\
\hline 285.48 & 2811.638 & 4496.303 & 4990.9602941 & 0.0078532 \\
656.20 & 972.896 & 4287.228 & 5773.4458560 & 0.0001212 \\
855.93 & 1882.429 & 3864.236 & 5689.5197041 & 0.0000769 \\
1547.09 & 12081.303 & 3028.602 & 6417.7371987 & 0.0001579 \\
\hline
\end{tabular}

Table 10 Parameters "A", "B" and "C" of the quadratic fit model (G2) are shown for each wavelength. Here, we can see that the quadratic adjustment improves to a certain extent the value of the estimated temperature of 2011-10-10.

\begin{tabular}{lllllr}
\hline $\begin{array}{l}\text { Wavelength } \\
{[\mathrm{nm}]}\end{array}$ & $\begin{array}{l}\text { Constant C } \\
{\left[\mathrm{K} /\left(\frac{\mathrm{W}}{\mathrm{m}^{2}} / \mathrm{nm}\right)^{2}\right]}\end{array}$ & $\begin{array}{l}\text { Constant A } \\
{\left[\mathrm{K} /\left(\frac{\mathrm{W}}{\mathrm{m}^{2}} / \mathrm{nm}\right)\right]}\end{array}$ & $\begin{array}{l}\text { Constant B } \\
{[\mathrm{K}]}\end{array}$ & $\begin{array}{l}\text { Estimated } T \\
{[\mathrm{~K}]}\end{array}$ & $\begin{array}{l}\text { Difference } \\
{[\mathrm{K}]}\end{array}$ \\
\hline 285.48 & -6395.976 & 5059.831 & 4298.750 & 4990.9681295 & 0.0000178 \\
656.20 & -161.712 & 1466.929 & 3909.908 & 5773.4459773 & 0.0000000 \\
855.93 & -398.428 & 2655.212 & 3489.518 & 5689.5197810 & 0.0000000 \\
1547.09 & -3829.317 & 14229.287 & 2727.384 & 6417.7373568 & -0.0000003 \\
\hline
\end{tabular}

$$
B^{\prime}=T_{o}-\left(\frac{d T}{d S S I}\right)_{o} * S S I_{o}+\frac{1}{2}\left(\frac{d^{2} T}{d S S I^{2}}\right)_{o} * S S I_{o}^{2}
$$

The values of constants $A^{\prime}, B^{\prime}, C^{\prime}$ in the analytic model, and $A, B, C$ in the fit model, are shown in Tables 8 and 10, along with values of $T$ obtained with the quadratic analytic model and the quadratic fit model for certain wavelengths.

As mentioned above, the linear fit is obtained with regression techniques in the $R$ software and for this, all the available spectral irradiance data are used for a fixed wavelength and therefore, if there is a change in the range of data, the linear fit changes because an analysis is done on all the data. In this way, a partition of the available data (2003 to 2020) into an "early period" designated R1 (2003 to 2010), and a "late period" designated R2 (2011 to 2020) was made to make a comparison between the linear coefficients shown in Tables 11 and 12 . 
Table 11 The constant $\boldsymbol{a}^{\prime}$ calculated using Equation G.5 or E.10, and the constant $\boldsymbol{a}$ calculated using Equation G.1 of the linear fit model, using $R$ software with data from 2003 to 2020.

\begin{tabular}{|c|c|c|c|c|}
\hline $\begin{array}{l}\text { Wavelength } \\
\text { [nm] }\end{array}$ & $\begin{array}{l}\text { Constant } \boldsymbol{a}^{\prime} \\
{\left[\mathrm{K} /\left(\mathrm{W} / \mathrm{m}^{2} / \mathrm{nm}\right)\right]}\end{array}$ & $\begin{array}{l}\text { Constant } \boldsymbol{a} \\
{\left[\mathrm{K} /\left(\mathrm{W} / \mathrm{m}^{2} / \mathrm{nm}\right)\right]}\end{array}$ & $\begin{array}{l}\text { Difference } \\
{\left[\mathrm{K} /\left(\mathrm{W} / \mathrm{m}^{2} / \mathrm{nm}\right)\right]}\end{array}$ & $\begin{array}{l}\text { Relative } \\
\text { Error } \\
{[\%]}\end{array}$ \\
\hline 358.48 & 873.388 & 873.145 & 0.243 & 0.03 \\
\hline 368.52 & 684.319 & 683.826 & 0.494 & 0.07 \\
\hline 378.65 & 671.005 & 670.186 & 0.819 & 0.12 \\
\hline 383.35 & 879.895 & 878.216 & 1.679 & 0.19 \\
\hline 390.26 & 700.309 & 699.454 & 0.855 & 0.12 \\
\hline 393.36 & 823.076 & 821.323 & 1.752 & 0.21 \\
\hline 410.70 & 560.720 & 560.594 & 0.126 & 0.02 \\
\hline 415.60 & 554.559 & 554.369 & 0.190 & 0.03 \\
\hline 420.68 & 566.168 & 566.304 & -0.135 & -0.02 \\
\hline 430.77 & 632.893 & 632.381 & 0.511 & 0.08 \\
\hline 434.31 & 583.383 & 583.232 & 0.151 & 0.03 \\
\hline 486.32 & 597.197 & 597.124 & 0.073 & 0.01 \\
\hline 527.52 & 655.268 & 655.235 & 0.033 & 0.01 \\
\hline 589.35 & 772.610 & 772.446 & 0.164 & 0.02 \\
\hline 619.44 & 855.672 & 855.470 & 0.202 & 0.02 \\
\hline 656.20 & 973.204 & 972.896 & 0.308 & 0.03 \\
\hline 758.01 & 1358.396 & 1358.008 & 0.388 & 0.03 \\
\hline 820.85 & 1670.225 & 1669.646 & 0.579 & 0.03 \\
\hline 855.93 & 1883.046 & 1882.429 & 0.616 & 0.03 \\
\hline 897.83 & 2128.196 & 2127.466 & 0.730 & 0.03 \\
\hline 1210.76 & 5263.981 & 5264.319 & -0.338 & -0.01 \\
\hline 1227.20 & 5493.583 & 5494.151 & -0.568 & -0.01 \\
\hline 1254.68 & 5910.298 & 5910.953 & -0.655 & -0.01 \\
\hline 1408.28 & 8702.579 & 8703.534 & -0.955 & -0.01 \\
\hline 1547.09 & 12080.859 & 12081.303 & -0.444 & 0.00 \\
\hline 1588.65 & 13326.562 & 13327.369 & -0.807 & -0.01 \\
\hline 1593.80 & 13477.502 & 13478.401 & -0.899 & -0.01 \\
\hline 1598.95 & 13630.355 & 13631.038 & -0.683 & -0.01 \\
\hline
\end{tabular}

\section{Appendix H: Example Calculations of Brightness Temperature Using the Analytic and Fit Models}

This appendix illustrates the $T$ approximations by considering an example of a randomly chosen day. For this example, results from the linear and quadratic analytic models are compared with the results of applying the linear and quadratic fit models for the randomly chosen day. In Tables 1-4, the RMSE (root-mean-square-error) and the ME (mean error, or bias), computed over all the available days in the SIM v27 record, are shown for all four models, linear and quadratic, analytic and fit.

To better explain how the analytic and fit models work, consider the following example for the wavelength of $\lambda=656.20 \mathrm{~nm}(\mathrm{H} \alpha)$. As mentioned above, the data of one particular day during solar minimum is taken as a reference, in this case 2008-08-24. On that 
Table 12 Values of the linear fit constant $\boldsymbol{a}$ calculated using two ranges of dates, R1 and R2: $\boldsymbol{a}$ R1 is the linear coefficient calculated with $R$ software using data from 2003 to 2010, while $a \mathrm{R} 2$ is calculated using data from 2011 to 2020 . The relative errors are also obtained when making the comparison with $\boldsymbol{a}^{\prime}$.

\begin{tabular}{|c|c|c|c|c|c|c|}
\hline $\begin{array}{l}\text { Wavelength } \\
{[\mathrm{nm}]}\end{array}$ & $\begin{array}{l}\text { Constant } \boldsymbol{a} \\
\text { R1 }\end{array}$ & $\begin{array}{l}\text { Constant } \boldsymbol{a} \\
\text { R2 }\end{array}$ & Diff. R1 & Diff. R2 & RE\% R1 & $\mathrm{RE} \% \mathrm{R} 2$ \\
\hline 358.48 & 872.602 & 874.854 & 0.787 & -1.466 & 0.09 & -0.17 \\
\hline 368.52 & 683.224 & 685.061 & 1.095 & -0.742 & 0.16 & -0.11 \\
\hline 378.65 & 669.821 & 670.869 & 1.183 & 0.135 & 0.18 & 0.02 \\
\hline 383.35 & 878.333 & 878.005 & 1.562 & 1.890 & 0.18 & 0.21 \\
\hline 390.26 & 699.234 & 699.953 & 1.076 & 0.356 & 0.15 & 0.05 \\
\hline 393.36 & 822.001 & 820.758 & 1.074 & 2.318 & 0.13 & 0.28 \\
\hline 410.70 & 560.697 & 560.571 & 0.024 & 0.149 & 0.00 & 0.03 \\
\hline 415.60 & 554.337 & 554.397 & 0.222 & 0.162 & 0.04 & 0.03 \\
\hline 420.68 & 566.357 & 566.278 & -0.189 & -0.109 & -0.03 & -0.02 \\
\hline 430.77 & 632.841 & 632.157 & 0.052 & 0.736 & 0.01 & 0.12 \\
\hline 434.31 & 583.339 & 583.147 & 0.044 & 0.237 & 0.01 & 0.04 \\
\hline 486.32 & 597.333 & 596.913 & -0.135 & 0.285 & -0.02 & 0.05 \\
\hline 527.52 & 655.425 & 655.043 & -0.156 & 0.226 & -0.02 & 0.03 \\
\hline 589.35 & 772.700 & 772.284 & -0.090 & 0.326 & -0.01 & 0.04 \\
\hline 619.44 & 855.733 & 855.319 & -0.061 & 0.353 & -0.01 & 0.04 \\
\hline 656.20 & 973.209 & 972.702 & -0.005 & 0.503 & 0.00 & 0.05 \\
\hline 758.01 & 1358.331 & 1357.818 & 0.064 & 0.578 & 0.00 & 0.04 \\
\hline 820.85 & 1669.971 & 1669.470 & 0.254 & 0.755 & 0.02 & 0.05 \\
\hline 855.93 & 1882.755 & 1882.294 & 0.291 & 0.751 & 0.02 & 0.04 \\
\hline 897.83 & 2128.273 & 2127.304 & -0.076 & 0.892 & 0.00 & 0.04 \\
\hline 1210.76 & 5264.415 & 5264.262 & -0.434 & -0.281 & -0.01 & -0.01 \\
\hline 1227.20 & 5493.734 & 5494.265 & -0.151 & -0.682 & 0.00 & -0.01 \\
\hline 1254.68 & 5910.018 & 5911.117 & 0.280 & -0.819 & 0.00 & -0.01 \\
\hline 1408.28 & 8701.740 & 8703.884 & 0.838 & -1.305 & 0.01 & -0.02 \\
\hline 1547.09 & 12076.345 & 12082.741 & 4.514 & -1.882 & 0.04 & -0.02 \\
\hline 1588.65 & 13323.272 & 13328.223 & 3.291 & -1.661 & 0.02 & -0.01 \\
\hline 1593.80 & 13474.719 & 13479.027 & 2.783 & -1.525 & 0.02 & -0.01 \\
\hline 1598.95 & 13627.132 & 13631.683 & 3.223 & -1.328 & 0.02 & -0.01 \\
\hline
\end{tabular}

"reference day", for the $\mathrm{H} \alpha$ wavelength, the solar spectral irradiance (SSI) according to the SIM instrument was, $S S I_{o}=1.526558 \mathrm{~W} \mathrm{~m}^{-2}(\mathrm{~nm})^{-1}$, which is the value registered in the data downloaded in LISIRD, in version 27. Using Equation D.2, checked with Mathematica root-finding software applied to Equation D.3, it was calculated from this reference-day irradiance that the exact brightness temperature is $T_{o}=5772.410671 \mathrm{~K}$. Note: We use high precision here in order to illustrate the very small RMSEs.

Next, using Equation D.3, divide $S S I_{o}$ by $\alpha_{\mathrm{s}}$ to compute $B_{o}=22468.34828$. Then using Equation E. 3 we compute $y_{o}=3.79838$. Substituting $y=y_{o}$, along with the $\mathrm{H} \alpha$ wavelength, and the two radiation constants $k_{1}$ and $k_{2}$, into Equation E. 10 we obtain

$$
\left(\frac{d T}{d S S I}\right)_{o}=973.20427 \mathrm{~K} * \frac{\mathrm{nm}}{\mathrm{W} / \mathrm{m}^{2}} .
$$


Table 13 Values of the quotients $\boldsymbol{a}^{\prime}$ and $\boldsymbol{a}$ for different wavelengths and the values of $\boldsymbol{a}^{\prime}$ obtained using the polynomial interpolation of Equations I.1 and I.2.

\begin{tabular}{|c|c|c|c|c|}
\hline $\begin{array}{l}\text { Wavelength } \\
{[\mathrm{nm}]}\end{array}$ & $\begin{array}{l}\text { Ratio } \boldsymbol{a}^{\prime} \\
{\left[\mathrm{K} / \mathrm{W} / \mathrm{m}^{2}\right]}\end{array}$ & $\begin{array}{l}\text { Ratio } a \\
{\left[\mathrm{~K} / \mathrm{W} / \mathrm{m}^{2}\right]}\end{array}$ & $\begin{array}{l}\operatorname{Diff}\left(\boldsymbol{a}^{\prime}-\boldsymbol{a}\right) \\
{\left[\mathrm{K} / \mathrm{W} / \mathrm{m}^{2}\right]}\end{array}$ & $\begin{array}{l}\text { Interpolation } \\
\text { Ratio }\left[\mathrm{K} / \mathrm{W} / \mathrm{m}^{2}\right]\end{array}$ \\
\hline 410.70 & 1.3653 & 1.3650 & 0.0003 & 1.3747 \\
\hline 415.60 & 1.3344 & 1.3339 & 0.0005 & 1.3694 \\
\hline 420.68 & 1.3458 & 1.3462 & -0.0003 & 1.3642 \\
\hline 430.77 & 1.4692 & 1.4680 & 0.0012 & 1.3548 \\
\hline 434.31 & 1.3432 & 1.3429 & 0.0003 & 1.3518 \\
\hline 486.32 & 1.2280 & 1.2278 & 0.0002 & 1.3251 \\
\hline 527.52 & 1.2422 & 1.2421 & 0.0001 & 1.3272 \\
\hline 589.35 & 1.3110 & 1.3107 & 0.0003 & 1.3688 \\
\hline 619.44 & 1.3814 & 1.3810 & 0.0003 & 1.4058 \\
\hline 656.20 & 1.4831 & 1.4826 & 0.0005 & 1.4658 \\
\hline 758.01 & 1.7921 & 1.7915 & 0.0005 & 1.7173 \\
\hline 820.85 & 2.0348 & 2.0340 & 0.0007 & 1.9351 \\
\hline 855.93 & 2.2000 & 2.1993 & 0.0007 & 2.0774 \\
\hline 897.83 & 2.3704 & 2.3696 & 0.0008 & 2.2669 \\
\hline 1210.76 & 4.3477 & 4.3479 & -0.0003 & 4.3532 \\
\hline 1227.20 & 4.4765 & 4.4770 & -0.0005 & 4.4955 \\
\hline 1254.68 & 4.7106 & 4.7111 & -0.0005 & 4.7407 \\
\hline 1408.28 & 6.1796 & 6.1803 & -0.0007 & 6.2794 \\
\hline 1547.09 & 7.8088 & 7.8090 & -0.0003 & 7.9152 \\
\hline 1588.65 & 8.3886 & 8.3891 & -0.0005 & 8.4503 \\
\hline 1593.80 & 8.4562 & 8.4568 & -0.0006 & 8.5180 \\
\hline 1598.95 & 8.5246 & 8.5250 & -0.0004 & 8.5861 \\
\hline 1601.18 & 8.5954 & 12.8930 & -4.2977 & 8.6157 \\
\hline 1616.86 & 8.8100 & 13.0572 & -4.2472 & 8.8254 \\
\hline 1678.61 & 9.7296 & 14.1889 & -4.4593 & 9.6800 \\
\hline 1768.43 & 11.2408 & 11.1965 & 0.0443 & 11.0054 \\
\hline
\end{tabular}

Similarly, using the same $B_{o}$ but varying wavelength, and so varying $y_{o}$, Table 6 shows the linear analytic model coefficients for the wavelengths of $285.50 \mathrm{~nm}, 656.20 \mathrm{~nm}$, $855.93 \mathrm{~nm}$ and $1547.09 \mathrm{~nm}$. The linear analytic approximation is used to estimate the value of $T$ for some other day, knowing the $S S I$ of that day. If we choose a random day, for example 2011-10-10, the value of SSI of that day (see Table 5) for the wavelength of $\mathrm{H} \alpha$ is $S S I=1.527622 \mathrm{Wm}^{-2}(\mathrm{~nm})^{-1}$. Using Equation E.18, without the quadratic term, then yields

$$
\begin{gathered}
T=5772.410671 \mathrm{~K}+973.20427 *(1.527622-1.526558) \mathrm{K} \\
T=5773.44616 \mathrm{~K} .
\end{gathered}
$$

This is the linear analytic approximation for the brightness temperature for the "example" date 2011-10-10. It is close to, but slightly larger than, the value computed by the "exact" Equation D.2 (or root finding in Mathematica software), $T=5773.44598 \mathrm{~K}$ (see Table 5). The error of root finding is very small compared to either the analytic or statistical estimates, for the relatively smooth functions involved here, so in this article both the result of using Equation D.2 and the root-finding result are referred to as the "exact" value. 
If we consider the root-mean-square-error (RMSE) in Table 2, the value obtained with the linear approximation, $5773.44616 \mathrm{~K} \pm 0.00041 \mathrm{~K}$, agrees very well with the exact value, since it is within the range of values. The exact value is obtained by applying Equation D.2. The approximate result of H.2 is obtained when using the Equation G.5 with the values of the constants in Table 7.

For the quadratic analytic approximation, we calculate the brightness temperature using Equation E.18, including the quadratic coefficient from Equations E.17, with D.3 and E.3, to give

$$
\left(\frac{d^{2} T}{d S S I^{2}}\right)_{o}=-323.64399 \mathrm{~K} *\left(\frac{\mathrm{nm}}{\mathrm{W} / \mathrm{m}^{2}}\right)^{2} .
$$

Similarly, the quadratic coefficients for the other wavelengths are given in Table 6 , but rounded to three places. Therefore, the value of the $\mathrm{H} \alpha$ temperature obtained by including the quadratic term in H.2 is $5773.44597715 \mathrm{~K}$, which when rounded to five places right of the decimal, agrees well with the exact. If we consider the RMSE, rounded to eight places, the range of the brightness temperature is $5773.44597715 \pm 0.00000034 \mathrm{~K}$, which also includes the exact value, and is much closer to the exact than is the linear.

The above was for a wavelength of $656.20 \mathrm{~nm}$. Table 5 shows the $S S I$ and $T$ values for this same wavelength, as well as for wavelengths of $285.50 \mathrm{~nm}, 855.93 \mathrm{~nm}$ and $1547.09 \mathrm{~nm}$, and also the SSI and exact $T$ values on 2011-10-10. Tables 7 and 8 show estimated results for 2011-10-10, using the analytic Equations G.5 and G.6, respectively.

Finally, in Tables 9 and 10 the parameters of linear G.1 and quadratic G.2 fit models are shown, and the value of the estimated brightness temperature on 2011-10-10. Comparing these results with the values of the RMSE and ME listed in Tables 1, 2, 3 and 4, the results are shown to be in excellent agreement with the exact values obtained from Equation D.2 or the Mathematica root-finding method.

\section{Appendix I: Temperature Sensitivity Ratios and Rapid Interpolation}

This final appendix provides a rapid method of interpolation between the measured wavelengths.

The ratio of a small change of the Sun's effective temperature divided by the associated change of the TSI is $1.06053 \mathrm{~K} /\left(\mathrm{W} / \mathrm{m}^{2}\right)$, as given by Equation F.4. The analogous spectral relationship is the linear coefficient $\boldsymbol{a}^{\prime}$ of the linear analytic approximation, the ratio of the change of spectral brightness temperature divided by the associated change in the SSI, the solar spectral irradiance, from the linear term in Equation E.18, using E.10 and D.3. To match the units of this TSI sensitivity ratio, it is appropriate to divide $\boldsymbol{a}^{\prime}$ by the wavelength $\lambda$. This allows determination of the wavelength for which the ratio $\boldsymbol{a}^{\prime} / \lambda$ is closest to the TSI value $1.06053 \mathrm{~K} /\left(\mathrm{W} / \mathrm{m}^{2}\right)$. The spectral values are given in Table 13, which shows a minimum value of $\frac{a^{\prime}}{\lambda}=1.2280$, which occurs near $486.3 \mathrm{~nm}$, whereas otherwise $\frac{a^{\prime}}{\lambda}>1.2280>$ $1.06053 \mathrm{~K} /\left(\mathrm{W} / \mathrm{m}^{2}\right)=$ TSI sensitivity.

For interpolation between measured wavelengths, it is useful to obtain a simple analytic mathematical expression for the brightness temperature sensitivity ratio $\boldsymbol{a}^{\prime} / \boldsymbol{\lambda}$. An interpolation function for the ratio $\frac{a^{\prime}}{\lambda}$ may be expressed as

$$
\frac{\boldsymbol{a}^{\prime}}{\lambda}=6.043791 \times 10^{-6} \lambda^{2}-6.076933 \times 10^{-3} \lambda+2.851032,
$$


where $\lambda$ is any wavelength that satisfies $400 \mathrm{~nm} \leq \lambda \leq 1800 \mathrm{~nm}$. With the previous expression one can calculate $\boldsymbol{a}^{\prime}$ for any wavelength $\lambda$ within this range, and then use the SSI value on any day to compute the associated brightness temperature $T$, using the linear analytic approximation, which can be written

$$
T=\boldsymbol{a}^{\prime} *\left(S S I-S S I_{o}\right)-T_{o} .
$$

Equation I.2 represents a method of calculating the brightness temperature that is simpler and faster than the linear term in Equation E.18, and valid for any wavelength within a broad range.

Acknowledgments We are grateful to Jerald Harder for support and encouragement, for his comments on an earlier version, and comments that improved this published version. We thank Stephane Beland for assistance obtaining the appropriate data from the LISARD system at University of Colorado's Laboratory for Atmospheric and Space Physics (LASP), and all those at LASP who designed and built the SORCE instruments and data system, and managed SORCE throughout the 17 years. We thank NASA Goddard Space Flight Center, the nonprofit CHEARS, and Universidad del Valle de Guatemala for sponsoring our research.

Data Availability The datasets generated during and/or analyzed during the current study are available from the corresponding author on reasonable request.

\section{Declarations}

Disclosure of Potential Conflicts of Interest The authors declare that they have no conflicts of interest.

Open Access This article is licensed under a Creative Commons Attribution 4.0 International License, which permits use, sharing, adaptation, distribution and reproduction in any medium or format, as long as you give appropriate credit to the original author(s) and the source, provide a link to the Creative Commons licence, and indicate if changes were made. The images or other third party material in this article are included in the article's Creative Commons licence, unless indicated otherwise in a credit line to the material. If material is not included in the article's Creative Commons licence and your intended use is not permitted by statutory regulation or exceeds the permitted use, you will need to obtain permission directly from the copyright holder. To view a copy of this licence, visit http://creativecommons.org/licenses/by/4.0/.

\section{References}

Adelberger, E.G., García, A., Robertson, R.G. Hamish, Snover, K.A., Balantekin, A.B., et al.: 2011, Solar fusion cross sections II: the pp chain and CNO cycles. Rev. Mod. Phys. 83, 195. DOI.

Bahcall, J.N.: 2000, How the Sun shines. J. Roy. Astron. Soc. Can. 94, 219. ADS.

Bethe, H.: 1939, Energy production in stars. Phys. Rev. 55, 436.

Eddy, J.A.: 2009, The Sun, the Earth and near-Earth Space: A guide to the Sun-Earth System. NP-20091066-GSFG, US Gov. Printing Office, Washington D.C. 1wstrt.gsfc.nasa.gov/images/pdf/john_eddy/ SES_Book_Interactive.pdf

Foukal, P., Lean, J.L.: 1985, The influence of faculae on total solar irradiance and luminosity. Astrophys. J. 302, 826. DOI.

Gray, L.J., Beer, J., Geller, M., Haigh, J.D., Lockwood, M., Matthes, K., et al.: 2010, Solar influences on climate. Rev. Geophys. 48, RG4001. DOI.

Harder, J.W.: 2020, In: SORCE SIM Level 3 Solar Spectral Irradiance Daily Means V027, Greenbelt, MD, USA, Goddard Earth Sciences Data and Information Services Center (GES DISC). DOI. Accessed: September 1, 2021.

Harder, J.W., Beland, S., Snow, M.: 2019, SORCE-based Solar Spectral Irradiance (SSI) record for input into chemistry-climate studies. Earth Space Sci. 6, 2487. DOI.

Harder, J.W., Lawrence, G., Fontenla, J., Rottman, G., Woods, T.: 2005, The spectral irradiance monitor: scientific requirements, instrument design, and operation modes. Solar Phys. 230, 141. DOI.

Harder, J.W., Fontenla, J.M., Pilewskie, P., Richard, E.C., Woods, T.N.: 2009, Trends in solar spectral irradiance variability in the visible and infrared. Geophys. Res. Lett. 36, L07801. DOI. 
Kopp, G.: 2016, Magnitudes and timescales of total solar irradiance variability. J. Space Weather Space Clim. 6, A30. DOI.

Kopp, G.: 2020, In: SORCE Level 3 Total Solar Irradiance Daily Means V019, Greenbelt, MD, USA, Goddard Earth Sciences Data and Information Services Center (GES DISC), DOI. Accessed: September 1, 2021.

Kopp, G., Lean, J.L.: 2011, A new, lower value of total solar irradiance: evidence and climate significance. Geophys. Res. Lett. 38, L01706. DOI.

Lee, J.N., Cahalan, R.F., Dong, L.W.: 2016, Solar rotational modulations of spectral irradiance and correlations with the variability of total solar irradiance. J. Space Weather Space Clim. 6, A33. DOI.

North, G., Cahalan, R., Coakley, J.: 1981, Energy balance climate models. Rev. Geophys. Space Phys. 19, 91.

Rottman, G., Cahalan, R.: 2002, SORCE: Solar Radiation and Climate Experiment, Goddard Space Flight Center, Greenbelt 24.

Shapiro, A.I., Solanki, S.K., Krivova, N.A., Tagirov, R.V., Schmutz, W.K.: 2015, The role of the Fraunhofer lines in solar brightness variability. Astron. Astrophys. 581, A116. DOI.

Shelyag, S., Schüssler, M., Solanki, S.K., Berdyugina, S.V., Vögler, A.: 2004, G-band spectral synthesis and diagnostics of simulated solar magneto-convection. Astron. Astrophys. 427, 335. DOI.

Solanki, S.K., Krivova, N.A., Haigh, J.D.: 2013, Solar irradiance variability and climate. Annu. Rev. Astron. Astrophys. 51, 311. DOI.

Stephens, G.L., O’Brien, D., Webster, P.J., Pilewski, P., Kato, S., Li, J.L: 2015, The albedo of Earth. Rev. Geophys. 53, 141. DOI.

Trishchenko, A.: 2005, Solar irradiance and effective brightness temperature for SWIR channels of AVHRR/NOAA and GOES images. J. Atmos. Ocean. Technol. 23, 198. DOI.

Wild, M., Folini, D., Schar, C., Loeb, N., Dutton, E.G., König-Langlo, G.: 2013, A new diagram of the global energy balance. AIP Conf. Proc. 1531, 628. DOI.

Wildt, R.: 1939, Electron affinity in astrophysics. Astrophys. J. 89, 295. DOI. ADS

Publisher's Note Springer Nature remains neutral with regard to jurisdictional claims in published maps and institutional affiliations. 